\title{
The flow and solidification of a thin fluid film on an arbitrary three-dimensional surface
}

\author{
T.G. Myers ${ }^{1}$, J.P.F. Charpin ${ }^{2}$, S.J. Chapman ${ }^{3}$
}

May 30, 2007

\begin{abstract}
A model for the flow of a thin film, with and without solidification, on an arbitrary threedimensional substrate is presented. The problem is reduced to two simultaneous partial differential equations for the film and solid layer thicknesses. The flow model (with the solidification rate set to zero) is the first such model to describe thin film flow on an arbitrary three-dimensional surface. Various limits are investigated to recover previous models for flow on flat, cylindrical and two-dimensional curved surfaces. With solidification a previous model for accretion on a flat substrate is retrieved. It is shown how the model may be reduced to standard forms, such as solidification on a flat surface, circular and non-circular cylinders, aerofoils and spheres. Numerical solutions are obtained by combining an ADI scheme with a shock capturing method. Results are presented for flow and accretion on a flat surface, aerofoil and sphere.
\end{abstract}

\footnotetext{
${ }^{1}$ Dept of Mathematics and Applied Mathematics, University of Cape Town, Rondebosch 7701, South Africa

${ }^{2}$ Applied Mathematics and Computing Group, Cranfield University, MK43 0AL, UK

${ }^{3}$ OCIAM, University of Oxford, Oxford OX1 3LB, UK
} 


\section{Introduction}

The flow of a thin fluid film with a single free surface has been the subject of intense investigation for a number of years, see $[1,2]$ for example. Recently a new level of complexity has been added to the problem by coupling the flow to a solidification model. Poots [3] has studied the steady flow of a water layer on an ice surface forming on a cylindrical power cable. Myers et al [4] study the unsteady flow of a solidifying fluid layer on a flat substrate, with the primary motive of predicting ice accretion on aircraft. The purpose of the current paper is to generalise such models. In the following a model will be developed to predict the flow and solidification of a thin fluid layer on an arbitrary three-dimensional surface. By setting the solid thickness to zero a general thin film flow model is obtained. This is the first general, three-dimensional curvilinear thin film flow model in the published literature.

The primary motivation for this work is the prediction of ice shapes on structures and aircraft. Ice accretion has been modelled for many years, following the pioneering work of Stefan $[5,6]$. An informative account of structural icing may be found in Poots [3]. Lock [7] deals with numerous forms of icing, including structural and in-flight. Structural icing is of particular interest in the US and Canada following the 'Great Ice Storm' of 1998 which caused billions of dollars of damage to power transmission and communication networks [8, 9]. Ice accretion has been shown to be the prime cause of a number of in-flight incidents and crashes and is therefore of ongoing concern to aircraft manufacturers throughout the world $[10,13]$. The work described in Myers et al is currently being used in a commercial aircraft icing code, ICECREMO $[11,12]$. An overview of aircraft icing may be found in $[10,11,13]$. Other processes involving the solidification of a thin flowing liquid layer include coating and spray forming. Coating has a vast number of applications, a number of references may be found in [1]. Spray forming, where an object is formed by the continual deposition of a fine spray, is studied in $[14,15]$.

Structural and aircraft icing typically occurs when supercooled droplets from clouds, freezing rain or drizzle impact on a cold surface. In very cold conditions the droplets freeze almost instantaneously to form rime ice. In this situation the ice growth rate is proportional 
to the amount of fluid impacting at each point, known as the 'catch' or 'collection efficiency'. Rime ice accretion is relatively well understood. In milder conditions a proportion of the impacting fluid can remain liquid for some time. This leads to glaze ice accretion. Since glaze accretion involves a liquid layer flowing on top of the ice surface, glaze ice shapes are significantly more difficult to predict than rime ice and consequently poorly understood.

In the following section, $\S 2$, the accretion and flow model is derived. Since the flow is the most complicated part of the model this is dealt with first in $§ 2.2$. The approximation employed to derive the flow model is not the standard lubrication approximation which requires both the square of the aspect ratio, $\epsilon^{2}$, and the reduced Reynolds number, $\epsilon^{2} R e$, to be small. In the following terms of $\mathcal{O}\left(\epsilon, \epsilon^{2} R e\right)$ will be neglected. This is not a significant restriction to lubrication theory since for many practical applications $\epsilon^{2} R e>\epsilon$ and so the leading order result is not affected. On the other hand, the derivation and expressions for fluid flux and velocities are significantly simplified by this approach. Dry accretion is dealt with in $§ 2.3 .1$. Wet accretion, which must be coupled to the flow model, is dealt with in $\S 2.3 .2$. In $\S 3$ the model is reduced to standard geometries: flat surface, cylinder and sphere. Numerical results are presented for each of these situations and also accretion on an aerofoil in $\S 4$.

\section{Governing equations for fluid flow and solidification $2.1 \quad$ Non-dimensionalisation}

The following derivation is in non-dimensional form, all variables are defined in the Nomenclature section. The non-dimensional variables are related to their dimensional counterparts (which are denoted by overbars) as follows:

$$
\begin{array}{lll}
\overline{s_{1}}=L s_{1} & \overline{s_{2}}=L s_{2} & \bar{\eta}=H \eta=\epsilon L \eta \\
\bar{u}=U u & \bar{v}=U v & \bar{w}=\epsilon U w \\
\bar{p}=P p & \overline{\mathbf{W}}=W \mathbf{W} \\
\bar{T}=\left(\bar{T}_{f}-\bar{T}_{s}\right) T+\bar{T}_{s} &
\end{array}
$$


where $H$ and $L$ represent height and length-scales. Note, this means that the dimensional co-ordinates must have the dimension of length. Care should be taken when the natural parameterisation involves an angle, for example in cylindrical co-ordinates $s_{1}=\theta$ is an obvious choice, but the correct choice in the current theory is the arc-length, $s_{1}=R \theta$. The aspect ratio $\epsilon=H / L \ll 1$. The time-scale

$$
\tau=\frac{\rho_{f} H}{\rho_{a} W}
$$

is determined by the rate at which fluid enters the system. In situations where there is no fluid impacting at the free surface, $\rho_{a} W=0$, the time-scale should be chosen according to the driving forces for the flow. However, the following theory remains the same. The local velocity of the droplets in the air is denoted $\overline{\mathbf{W}}$, this is scaled with a typical value $W$, the fluid velocity scale $U=L / \tau$.

\subsection{Fluid flow}

Figure 1 near here

The problem configuration is shown on Figure 1. Incoming fluid impacts on the substrate. An accretion layer of thickness $b$ forms, on top of this a thin fluid film of thickness $h$ may be present. The accretion temperature is denoted $T$, the fluid temperature $\chi$. The substrate is defined by $\mathbf{r}=\mathbf{R}\left(s_{1}, s_{2}\right)$, where $\left(s_{1}, s_{2}\right)$ are orthogonal surface co-ordinates. Provided the accretion or fluid layer is thin, a point in the accretion or fluid layer may be defined by

$$
\mathbf{r}=\mathbf{R}\left(s_{1}, s_{2}\right)+\epsilon \eta \mathbf{n}\left(s_{1}, s_{2}\right)
$$

where $\epsilon \ll 1$ is the aspect ratio, $\eta$ is the co-ordinate orthogonal to the substrate and $\mathbf{n}$ is the unit normal. To determine the derivatives in the governing equations the scaling factors $h_{1}, h_{2}, h_{3}$ must first be determined. These are defined as

$$
\begin{aligned}
h_{1}^{2} & =\frac{\partial \mathbf{r}}{\partial s_{1}} \cdot \frac{\partial \mathbf{r}}{\partial s_{1}}=E\left(1-\epsilon \eta \kappa_{1}\right)^{2}, \\
h_{2}^{2} & =\frac{\partial \mathbf{r}}{\partial s_{2}} \cdot \frac{\partial \mathbf{r}}{\partial s_{2}}=G\left(1-\epsilon \eta \kappa_{2}\right)^{2} \\
h_{3}^{2} & =\frac{\partial \mathbf{r}}{\partial \eta} \cdot \frac{\partial \mathbf{r}}{\partial \eta}=\epsilon^{2},
\end{aligned}
$$


where the first fundamental forms are

$$
E=\frac{\partial \mathbf{R}}{\partial s_{1}} \cdot \frac{\partial \mathbf{R}}{\partial s_{1}} \quad F=\frac{\partial \mathbf{R}}{\partial s_{1}} \cdot \frac{\partial \mathbf{R}}{\partial s_{2}}=0 \quad G=\frac{\partial \mathbf{R}}{\partial s_{2}} \cdot \frac{\partial \mathbf{R}}{\partial s_{2}}
$$

and $F=0$ since $\left(s_{1}, s_{2}\right)$ are orthogonal. The curvatures are defined as

$$
\begin{aligned}
& \kappa_{1}=-\frac{\partial \mathbf{R}}{\partial s_{1}} \cdot \frac{\partial \mathbf{n}}{\partial s_{1}}\left(\frac{\partial \mathbf{R}}{\partial s_{1}} \cdot \frac{\partial \mathbf{R}}{\partial s_{1}}\right)^{-1}=\frac{\partial^{2} \mathbf{R}}{\partial s_{1}{ }^{2}} \cdot \mathbf{n}\left(\frac{\partial \mathbf{R}}{\partial s_{1}} \cdot \frac{\partial \mathbf{R}}{\partial s_{1}}\right)^{-1}=\frac{L}{E} \\
& \kappa_{2}=-\frac{\partial \mathbf{R}}{\partial s_{2}} \cdot \frac{\partial \mathbf{n}}{\partial s_{2}}\left(\frac{\partial \mathbf{R}}{\partial s_{2}} \cdot \frac{\partial \mathbf{R}}{\partial s_{2}}\right)^{-1}=\frac{\partial^{2} \mathbf{R}}{\partial s_{2}{ }^{2}} \cdot \mathbf{n}\left(\frac{\partial \mathbf{R}}{\partial s_{2}} \cdot \frac{\partial \mathbf{R}}{\partial s_{2}}\right)^{-1}=\frac{N}{G} .
\end{aligned}
$$

The second fundamental forms are

$$
L=\mathbf{n} \cdot \frac{\partial^{2} \mathbf{R}}{\partial s_{1}{ }^{2}} \quad M=\mathbf{n} \cdot \frac{\partial^{2} \mathbf{R}}{\partial s_{1} s_{2}}=0 \quad N=\mathbf{n} \cdot \frac{\partial^{2} \mathbf{R}}{\partial s_{2}{ }^{2}},
$$

where $M=0$ since $\left(s_{1}, s_{2}\right)$ are geodesic co-ordinates. In deriving (6), (7) and $M=0$ the derivatives of

$$
\frac{\partial \mathbf{R}}{\partial s_{1}} \cdot \mathbf{n}=0=\frac{\partial \mathbf{R}}{\partial s_{2}} \cdot \mathbf{n}
$$

with respect to $s_{1}$ and $s_{2}$ have been used.

With the standard lubrication approximation, as well as neglecting terms of order $\epsilon \kappa$, the components of the Navier-Stokes equation may now be written as

$$
\begin{aligned}
\frac{\partial^{2} u}{\partial \eta^{2}} & =\frac{1}{E^{1 / 2}} \frac{\partial p}{\partial s_{1}}-B \mathbf{g} \cdot \mathbf{e}_{1}+\mathcal{O}\left(\epsilon, \epsilon^{2} R e\right) \\
\frac{\partial^{2} v}{\partial \eta^{2}} & =\frac{1}{G^{1 / 2}} \frac{\partial p}{\partial s_{2}}-B \mathbf{g} \cdot \mathbf{e}_{2}+\mathcal{O}\left(\epsilon, \epsilon^{2} R e\right) \\
\frac{\partial p}{\partial \eta} & =\epsilon B \mathbf{g} \cdot \mathbf{n}+\mathcal{O}\left(\epsilon, \epsilon^{2} R e\right)
\end{aligned}
$$

where the scale-factors $h_{i}$ have been used to determine the derivatives. The unit vectors in the $\left(s_{1}, s_{2}\right)$ directions are

$$
\mathbf{e}_{1}=\frac{1}{E^{1 / 2}} \frac{\partial \mathbf{R}}{\partial s_{1}} \quad \mathbf{e}_{2}=\frac{1}{G^{1 / 2}} \frac{\partial \mathbf{R}}{\partial s_{2}}
$$

and the normal $\mathbf{n}=\mathbf{e}_{1} \times \mathbf{e}_{2}$. The Bond number $B=\epsilon^{2} \rho_{f} g L^{2} /(\mu U)$, is the ratio between gravity and viscous forces. The term $\epsilon B$ is retained in the leading order balance (10) since 
the value of $B$ is, as yet, undetermined. This gravity term will drive the flow only when all other forces are $\mathcal{O}(\epsilon)$, i.e. when the substrate is almost horizontal over a significant region and surface forces are negligible. In this case the correct choice of velocity scale is $U=\epsilon^{3} \rho_{f} g L^{2} / \mu$ and gravity balances pressure gradient in equation (10). However, since this case is a simple and limited extension of the present analysis (which has also been covered previously [4]) the gravity term in equation (10) will be neglected from now on. For an incompressible fluid the continuity equation is

$$
\frac{\partial}{\partial s_{1}}\left(G^{1 / 2} u\right)+\frac{\partial}{\partial s_{2}}\left(E^{1 / 2} v\right)+\frac{\partial}{\partial \eta}\left(E^{1 / 2} G^{1 / 2} w\right)=0
$$

Equations $(8)-(10),(12)$ require solving subject to the following boundary conditions. On the accreting surface, $\eta=b$, there is no slip

$$
u=v=0
$$

At the free surface, $\eta=b+h$, there is continuity of shear and normal stresses

$$
A_{1}=\frac{\partial u}{\partial \eta}, \quad \quad A_{2}=\frac{\partial v}{\partial \eta}, \quad p-p_{0}=C^{\prime} \sigma \kappa^{\prime}
$$

where $A_{i}=h_{0} \bar{A}_{i} /(\mu U)$ denotes the non-dimensional shear stress. The shear could be due to a constant air shear [4, 18], or surface tension gradient [16, 17, 28]. The normal stress condition, (14), involves the ambient pressure, $p_{0}$, the mean curvature at the free surface, $\kappa^{\prime}$, and an inverse capillary number, $C^{\prime}=\epsilon^{2} \sigma_{0} / \mu U$ which represents the ratio between surface tension and viscous forces. It is denoted $C^{\prime}$ to distinguish it from the standard inverse capillary number $C=\epsilon^{3} \sigma_{0} / \mu U$. With certain fluids the dimensional surface tension $\bar{\sigma}=\sigma_{0} \sigma\left(s_{1}, s_{2}\right)$ may vary with position, in which case the shear stress components, $A_{i}$, represent the surface tension gradient. When dealing with derivatives of pressure the derivatives of surface tension must also be calculated, see [28] for example. When the surface tension is constant $\sigma \equiv 1$. The mean curvature at $\mathbf{r}=\mathbf{R}+\epsilon(b+h) \mathbf{n}$ is the sum of the principal curvatures in the $s_{1}, s_{2}$ directions, $\kappa^{\prime}=\kappa_{1}^{\prime}+\kappa_{2}^{\prime}$. The principal curvatures are the eigenvalues of

$$
\left(\begin{array}{c}
L^{\prime} M^{\prime} \\
M^{\prime} N^{\prime}
\end{array}\right)-\lambda\left(\begin{array}{cc}
E^{\prime} F^{\prime} \\
F^{\prime} G^{\prime}
\end{array}\right)=0
$$


where

$$
\begin{gathered}
E^{\prime}=E\left(1-\epsilon(b+h) \kappa_{1}\right)^{2}+\mathcal{O}\left(\epsilon^{2}\right), \quad F^{\prime}=\mathcal{O}\left(\epsilon^{2}\right), \quad G^{\prime}=G\left(1-\epsilon(b+h) \kappa_{2}\right)^{2}+\mathcal{O}\left(\epsilon^{2}\right) \\
L^{\prime}=L+\epsilon \frac{\partial^{2}}{\partial s_{1}{ }^{2}}(b+h)-\epsilon(b+h) \kappa_{1}^{2} E, \quad M^{\prime}=O(\epsilon), \\
N^{\prime}=N+\epsilon \frac{\partial^{2}}{\partial s_{2}{ }^{2}}(b+h)-\epsilon(b+h) \kappa_{2}^{2} G .
\end{gathered}
$$

Since $M^{\prime}=\mathcal{O}(\epsilon), F^{\prime}=\mathcal{O}\left(\epsilon^{2}\right)$ the curvatures are simply

$$
\begin{aligned}
\kappa_{1}^{\prime} & =\frac{L^{\prime}}{E^{\prime}}+\mathcal{O}\left(\epsilon^{2}\right)=\kappa_{1}+\epsilon \kappa_{1}^{2}(b+h)+\frac{\epsilon}{E} \frac{\partial^{2}}{\partial s_{1}^{2}}(b+h)+\mathcal{O}\left(\epsilon^{2}\right) \\
\kappa_{2}^{\prime} & =\frac{N^{\prime}}{G^{\prime}}+\mathcal{O}\left(\epsilon^{2}\right)=\kappa_{2}+\epsilon \kappa_{2}^{2}(b+h)+\frac{\epsilon}{G} \frac{\partial^{2}}{\partial s_{2}^{2}}(b+h)+\mathcal{O}\left(\epsilon^{2}\right) .
\end{aligned}
$$

Hence

$$
\begin{aligned}
p & =p_{0}+C^{\prime} \sigma \kappa^{\prime} \\
& =p_{0}-C^{\prime} \sigma\left(\kappa_{1}+\kappa_{2}+\epsilon(b+h)\left(\kappa_{1}^{2}+\kappa_{2}^{2}\right)+\epsilon\left[\frac{1}{E} \frac{\partial^{2}}{\partial s_{1}{ }^{2}}(b+h)+\frac{1}{G} \frac{\partial^{2}}{\partial{s_{2}}^{2}}(b+h)\right]\right)+\mathcal{O}\left(\epsilon^{2}\right) .
\end{aligned}
$$

When the flow is driven by pressure gradient it is clear from (18) that, whenever the substrate curvature is non-constant and $\mathcal{O}(1)$ it is the substrate curvature, $\kappa_{1}+\kappa_{2}$, that dominates the surface tension terms and therefore drives the flow. In this case the final terms of $\mathcal{O}\left(\epsilon C^{\prime}\right)$ in equation (18) should be neglected. When the mean substrate curvature is small, $\kappa_{1}+\kappa_{2} \sim \mathcal{O}(\epsilon)$, the terms involving the accretion and fluid heights become important and the standard inverse capillary number $C=\epsilon C^{\prime}$ is recovered. This occurs, for example, on an almost flat substrate, see $§ 3.1$. Alternatively if the mean substrate curvature is approximately constant, $\kappa_{1}+\kappa_{2} \sim$ constant $+\mathcal{O}(\epsilon)$, such as occurs on an almost circular cylinder or spherical substrate (see $\S 3.2,3.4$ ) then all of the surface tension terms in the pressure gradient are again $\mathcal{O}\left(\epsilon C^{\prime}\right)$.

At the accretion-fluid and fluid-air interfaces there is continuity of mass flux:

$$
\rho_{1} \mathbf{n}^{\prime} \cdot\left(\mathbf{u}_{1}-\mathbf{u}_{\mathbf{b}}\right)=\rho_{2} \mathbf{n}^{\prime} \cdot\left(\mathbf{u}_{2}-\mathbf{u}_{\mathbf{b}}\right)
$$

where $\rho_{k}$ and $\mathbf{u}_{\mathbf{k}}$ are the density and velocity in each phase and $\mathbf{u}_{\mathbf{b}}$ is the velocity of the boundary between the two phases, $\mathbf{n}^{\prime}$ is the normal to the surface. The normal at the 
accretion-fluid interface is

$$
\mathbf{n}^{\prime}=\left(\frac{\partial \mathbf{r}}{\partial s_{1}} \times \frac{\partial \mathbf{r}}{\partial s_{2}}\right)\left|\frac{\partial \mathbf{r}}{\partial s_{1}} \times \frac{\partial \mathbf{r}}{\partial s_{2}}\right|^{-1}
$$

where

$$
\frac{\partial \mathbf{r}}{\partial s_{1}}=\frac{\partial \mathbf{R}}{\partial s_{1}}+\epsilon b \frac{\partial \mathbf{n}}{\partial s_{1}}+\epsilon \frac{\partial b}{\partial s_{1}} \mathbf{n}=\left(1-\epsilon b \kappa_{1}\right) \frac{\partial \mathbf{R}}{\partial s_{1}}+\epsilon \frac{\partial b}{\partial s_{1}} \mathbf{n}
$$

with a similar expression for $\partial \mathbf{r} / \partial s_{2}$. Therefore

$$
\mathbf{n}^{\prime}=\mathbf{n}-\frac{\epsilon}{E^{1 / 2}} \frac{\partial b}{\partial s_{1}} \mathbf{e}_{1}-\frac{\epsilon}{G^{1 / 2}} \frac{\partial b}{\partial s_{2}} \mathbf{e}_{2}+\mathcal{O}\left(\epsilon^{2}\right)
$$

The values for the velocities at the accretion-fluid interface, required in (19), are

$$
\mathbf{u}_{b}=\frac{\partial \mathbf{r}}{\partial t}=\epsilon \frac{\partial b}{\partial t} \mathbf{n} \quad \mathbf{u}_{1}=(0,0, \epsilon w) \quad \mathbf{u}_{2}=(0,0,0)
$$

where $\mathbf{u}_{1}$ is the fluid velocity (which is zero in the $s_{1}, s_{2}$ directions due to the no-slip condition), $\mathbf{u}_{2}$ is the solid velocity and $\rho_{1}=\rho_{f}, \rho_{2}=\rho_{s}$. To leading order in $\epsilon$ the normal velocity condition at $\eta=b$ is

$$
w=\left(1-\frac{\rho_{s}}{\rho_{f}}\right) \frac{\partial b}{\partial t}
$$

If the fluid and solid densities are the same then $w=0$ on $\eta=b$. Normal fluid motion only occurs if there is a density difference and the fluid must move to accommodate the new solid.

At the air-fluid interface the normal is

$$
\mathbf{n}^{\prime}=\mathbf{n}-\frac{\epsilon}{E^{1 / 2}} \frac{\partial}{\partial s_{1}}(b+h) \mathbf{e}_{1}-\frac{\epsilon}{G^{1 / 2}} \frac{\partial}{\partial s_{2}}(b+h) \mathbf{e}_{2}+\mathcal{O}\left(\epsilon^{2}\right)
$$

and the velocities are

$$
\mathbf{u}_{b}=\frac{\partial \mathbf{r}}{\partial t}=\epsilon \frac{\partial}{\partial t}(b+h) \mathbf{n} \quad \mathbf{u}_{1}=\epsilon \frac{\rho_{f}}{\rho_{a}}\left(\mathbf{W} \cdot \mathbf{e}_{\mathbf{1}}, \mathbf{W} \cdot \mathbf{e}_{\mathbf{2}}, \mathbf{W} \cdot \mathbf{n}\right) \quad \mathbf{u}_{2}=(u, v, \epsilon w)
$$

where $\mathbf{u}_{\mathbf{1}}, \mathbf{u}_{\mathbf{2}}$ now represent the air (droplet) and fluid velocities respectively. The velocity of the incoming fluid is denoted W. As with gravity this will be expressed in an external (as opposed to surface) co-ordinate system. The density of the fluid in the air is denoted $\rho_{a}$. The factor $\epsilon \rho_{f} / \rho_{a}$ occurs due to the chosen time-scale. In dimensional form the velocity 
$\mathbf{u}_{1}$ may be expressed as $U \mathbf{u}_{1}=W\left(\mathbf{W} \cdot \mathbf{e}_{\mathbf{1}}, \mathbf{W} \cdot \mathbf{e}_{\mathbf{2}}, \mathbf{W} \cdot \mathbf{n}\right)$, where $U=L / \tau$ and according to equation (1), $W=\rho_{f} H /\left(\rho_{a} \tau\right)$, hence $W / U=\epsilon \rho_{f} / \rho_{a}$. The density of fluid in the air stream is, in practical situations, significantly less than the density of the fluid film, $\rho_{a} \ll \rho_{f}$. In the following $\rho_{a} / \rho_{f}$ will be neglected with respect to $\mathcal{O}(1)$ terms. To leading order in $\epsilon$ the normal velocity condition at $\eta=b+h$ is therefore

$$
w=\left(\frac{\partial b}{\partial t}+\frac{\partial h}{\partial t}\right)+\mathbf{W} \cdot \mathbf{n}+\frac{1}{E^{1 / 2}} u \frac{\partial}{\partial s_{1}}(b+h)+\frac{1}{G^{1 / 2}} v \frac{\partial}{\partial s_{2}}(b+h) .
$$

The pressure is determined by integrating (10) subject to (18)

$$
p=p_{0}-C^{\prime} \sigma\left(\kappa_{1}+\kappa_{2}+\epsilon(b+h)\left(\kappa_{1}^{2}+\kappa_{2}^{2}\right)+\epsilon\left[\frac{1}{E} \frac{\partial^{2}}{\partial s_{1}^{2}}(b+h)+\frac{1}{G} \frac{\partial^{2}}{\partial s_{2}{ }^{2}}(b+h)\right]\right) \text {. }
$$

The $\mathcal{O}\left(\epsilon C^{\prime}\right)$ terms are retained in this expression so that flows over a constant curvature surface may be modelled. Since it is pressure gradient, rather than pressure, which may drive a flow, when $\kappa_{1}, \kappa_{2}$ are constant the driving force consists solely of the $\mathcal{O}\left(\epsilon C^{\prime}\right)$ terms. The driving force is then $\mathcal{O}(C)$ where $C=\epsilon C^{\prime}$ is the standard capillary number. The velocities are determined by integrating (8), (9) subject to (13), (14)

$$
\begin{aligned}
& u=\left(\frac{1}{E^{1 / 2}} \frac{\partial p}{\partial s_{1}}-B \mathbf{g} \cdot \mathbf{e}_{\mathbf{1}}\right)\left(\frac{\eta^{2}-b^{2}}{2}-(\eta-b)(b+h)\right)+A_{1}(\eta-b) \\
& v=\left(\frac{1}{G^{1 / 2}} \frac{\partial p}{\partial s_{2}}-B \mathbf{g} \cdot \mathbf{e}_{\mathbf{2}}\right)\left(\frac{\eta^{2}-b^{2}}{2}-(\eta-b)(b+h)\right)+A_{2}(\eta-b) .
\end{aligned}
$$

As discussed in the introduction terms of $\mathcal{O}(\epsilon)$ have been neglected in deriving this expression. However, it is a simple matter to integrate the velocity equations whilst retaining $\mathcal{O}(\epsilon)$ terms. The velocity expressions then involve logarithms of $\eta$. In the limit $\epsilon \rightarrow 0$ equations (29), (30) are retrieved. Weidner et al [27] have used the logarithmic form in a study of flow on a cylinder.

Integrating the continuity equation across the film gives

$$
\begin{aligned}
\left.w\right|_{b+h}-\left.w\right|_{b}= & -\frac{1}{(E G)^{1 / 2}} \int_{b}^{b+h} \frac{\partial}{\partial s_{1}}\left(u G^{1 / 2}\right)+\frac{\partial}{\partial s_{2}}\left(v E^{1 / 2}\right) d \eta \\
= & -\frac{1}{(E G)^{1 / 2}}\left(\frac{\partial}{\partial s_{1}} \int_{b}^{b+h} u G^{1 / 2} d \eta+\frac{\partial}{\partial s_{2}} \int_{b}^{b+h} v E^{1 / 2} d \eta\right) \\
& \quad+\left.\frac{1}{E^{1 / 2}} u\right|_{b+h} \frac{\partial}{\partial s_{1}}(b+h)+\left.\frac{1}{G^{1 / 2}} v\right|_{b+h} \frac{\partial}{\partial s_{2}}(b+h) .
\end{aligned}
$$


Substituting for $w$ via (24), (27) and evaluating the integrals leads to

$$
\frac{\partial h}{\partial t}+\nabla_{s} \cdot \mathbf{Q}=-\frac{\rho_{s}}{\rho_{f}} \frac{\partial b}{\partial t}-\mathbf{W} \cdot \mathbf{n}
$$

where $\nabla_{s}$ represents the surface operator,

$$
\nabla_{s} \cdot \mathbf{Q}=\frac{1}{(E G)^{1 / 2}}\left(G^{1 / 2} \frac{\partial}{\partial s_{1}} Q_{1}+E^{1 / 2} \frac{\partial}{\partial s_{2}} Q_{2}\right)
$$

and

$$
\begin{aligned}
& Q_{1}=\int_{b}^{b+h} u d \eta=-\left(\frac{1}{E^{1 / 2}} \frac{\partial p}{\partial s_{1}}-B \mathbf{g} \cdot \mathbf{e}_{\mathbf{1}}\right) \frac{h^{3}}{3}+A_{1} \frac{h^{2}}{2} \\
& Q_{2}=\int_{b}^{b+h} v d \eta=-\left(\frac{1}{G^{1 / 2}} \frac{\partial p}{\partial s_{2}}-B \mathbf{g} \cdot \mathbf{e}_{2}\right) \frac{h^{3}}{3}+A_{2} \frac{h^{2}}{2}
\end{aligned}
$$

Equation (32) is the governing equation for the fluid flow. It demonstrates that the film thickness varies due to fluid flux, solidification rate and the rate at which fluid enters the system. The flux terms (34), (35) show that the fluid is driven by pressure gradient, gravity (along the surface) and surface shear. Provided the substrate is not horizontal everywhere, the pressure gradient depends on the ambient pressure (which may vary significantly around an aerofoil for example) and surface tension. Equation (32) involves two unknowns, the film height $h$ and the solid height $b$. A second equation to close the sytem will be derived in the following section via an energy balance. In the absence of solidification, $b \equiv 0$, equation (32) alone (subject to appropriate boundary conditions) is sufficient to predict the evolution of a thin fluid layer on an arbitrary three-dimensional surface. It is then a general form of the fourth-order, nonlinear degenerate parabolic partial differential equation typical of thin film, free surface flows $[1,2]$.

\subsection{Solidification}

The energy balance is derived subject to the following assumptions:

1. The Peclet number, $P e \ll 1$.

2. The previous approximations hold, $\epsilon, \epsilon^{2} R e \ll 1$. 
Assumption 1 means that the energy transfer across the layers is driven by conduction rather than advection. Assumption 2 may be strengthened to the standard level for lubrication approximation with $\epsilon^{2} \ll 1$ without affecting the following analysis. It will also be assumed that there is perfect thermal contact between the accretion and substrate and that the substrate temperature is constant. This means that the substrate has a high thermal mass and conductivity. A consequence of this restriction is that a proportion of the initial incoming fluid must solidify. Fluid flow may only occur once a solid layer exists to insulate the fluid layer from the substrate. With the current non-dimensionalisation the appropriate boundary condition is $T=0$ at $\eta=0$. Obviously it is a simple matter to adapt this to an imperfect thermal contact and a variable substrate temperature by choosing a cooling condition. For one-dimensional ice accretion this is considered in Myers \& Hammond [19].

Due to this final assumption the accretion occurs in two stages. First, dry accretion occurs. In this case the accretion shape is completely determined by a mass balance. After a certain amount of time fluid flows over the accretion. The problem is then governed by combined mass and energy balances.

\subsubsection{Dry accretion}

Initially there is no fluid flow and all terms on the left hand side of the mass balance (32) are identically zero. The solid height is therefore given by

$$
\frac{\partial b}{\partial t}=-\frac{\rho_{f}}{\rho_{s}} \mathbf{W} \cdot \mathbf{n} \text {. }
$$

In general the air flow varies with space and time and the solution of (36) must be determined numerically. However, if the air flow and droplet trajectories remain constant in time it may be integrated immediately

$$
b=-\frac{\rho_{f}}{\rho_{s}} \mathbf{W} \cdot \mathbf{n} t .
$$

Fluid will first appear when the accretion temperature reaches the freezing temperature. To determine when this occurs the thermal problem must be analysed. The temperature is specified by

$$
P e \frac{\partial T}{\partial t}=\nabla^{2} T
$$


where $P e=\epsilon^{2} \rho_{s} c_{s} L^{2} /\left(k_{s} \tau\right)$ is the Peclet number. In the current study the time-scale is determined by the rate at which fluid enters the system, $\tau=\rho_{f} H / \rho_{a} W$, and $P e=$ $\rho_{s} \rho_{a} W H c_{s} / k_{s} \rho_{f}$. In atmospheric icing calculations $\rho_{a} \approx 10^{-3} \mathrm{~kg} / \mathrm{m}^{3}$ is a typical liquid water content of the air, $W \approx[1,100] \mathrm{m} / \mathrm{s}$ is the velocity of the air flow (the lower limit applies to structural icing, the upper to aircraft icing), $H \approx 0.1 \mathrm{~mm}$ for aircraft icing and $1 \mathrm{~mm}$ for structural icing, $c_{s} \approx 2050 \mathrm{~J} / \mathrm{kg} \mathrm{K}, k_{s} \approx 2.18 \mathrm{~W} / \mathrm{m} \mathrm{K}$. The Peclet number $P e \in\left[10^{-4}, 10^{-2}\right]$ is small and therefore may be neglected. The same approximation will hold for other physically realistic situations however, care should be taken to ensure $P e \ll 1$ before applying the following approximations. Provided $P e \ll 1$ equation (38) reduces to a quasi-steady form at leading order:

$$
\frac{\partial^{2} T}{\partial \eta^{2}}=0
$$

This requires solving subject to the following conditions. At the free surface $\eta=b$ a heat flux condition holds:

$$
\left.\frac{\partial T}{\partial \eta}\right|_{\eta=b}=E_{0 D}-E_{1 D} T .
$$

The energy terms $E_{0 D}$ and $E_{1 D}$ can incorporate quantities such as latent heat, kinetic energy, radiation, conduction and convection. If the air flow varies with time the energy terms will also be time dependent. Details of these terms for structural and aircraft icing may be found in $[3,4,13,21]$. As discussed, at the solid substrate, $\eta=0$, there is continuity of temperature:

$$
T=0
$$

The appropriate solution of (39) is

$$
T=\frac{E_{0 D}}{1+E_{1 D} b} \eta
$$

Fluid first appears when the top of the layer, $\eta=b$, reaches the fusion temperature $T=1$. The corresponding accretion thickness, $b_{f}$, may be determined by solving (42) with $T=1$ to give

$$
b_{f}=\frac{1}{E_{0 D}-E_{1 D}} .
$$


If accretion occurs for a sufficiently short time, so that $b$ is never greater than $b_{f}$, then fluid will not appear. It is also possible that the ambient conditions are such that fluid will never appear, i.e. if $E_{0}-E_{1} \leq 0$. In either case the accretion shape is determined by (36) or (37). The temperature is given by (42) and the problem is completely solved.

\subsubsection{Wet accretion}

Once the accretion surface has reached the fusion temperature fluid will appear on the surface. The mass balance (32) involves two unknowns, the accretion and film heights, $b$ and $h$. To close the system an energy balance is required.

At the accretion-fluid interface, $\eta=b$, the energy balance is

$$
\left[\rho_{s} \mathcal{E}_{s}\left(\mathbf{u}_{\mathbf{s}}-\mathbf{u}_{\mathbf{b}}\right)-\rho_{f} \mathcal{E}_{f}\left(\mathbf{u}-\mathbf{u}_{\mathbf{b}}\right)\right] \cdot \mathbf{n}^{\prime}=\frac{\tau\left(\bar{T}_{f}-\bar{T}_{s}\right)}{L^{2}}\left[\nabla\left(k_{s} T\right)-\nabla\left(k_{f} \chi\right)\right] \cdot \mathbf{n}^{\prime},
$$

where $\mathcal{E}$ is the enthalpy of each phase and $\chi$ is the fluid temperature. The velocities are specified by equation (23) and the normal on the accreting substrate by (22). The gradient operator is defined by $\nabla=\left(1 / E^{1 / 2} \partial / \partial s_{1}, 1 / G^{1 / 2} \partial / \partial s_{2}, 1 / \epsilon \partial / \partial \eta\right)$. Equation (44) states that the energy created during the phase change is conducted away from the interface either through the accretion or the fluid layer. Substituting for the velocities and expanding (44) leads to

$$
S \frac{\partial b}{\partial t}=\frac{\partial T}{\partial \eta}-\frac{k_{f}}{k_{s}} \frac{\partial \chi}{\partial \eta}+\mathcal{O}\left(\epsilon^{2}\right)
$$

The Stefan number, $S=\left(\rho_{s} L_{f} H^{2}\right) /\left(\tau k_{s}\left(\bar{T}_{f}-\bar{T}_{s}\right)\right)$, is the ratio between the phase change energy and the conductive energy. The latent heat of fusion is defined as the jump in enthalpy $L_{f}=\mathcal{E}_{f}-\mathcal{E}_{s}$.

To solve equation (45), expressions for the ice and water temperature gradients are required. Again, provided $P e \ll 1$, the leading order heat equations simplify to quasi-steady forms:

$$
\frac{\partial^{2} T}{\partial \eta^{2}}=0 \quad \frac{\partial^{2} \chi}{\partial \eta^{2}}=0
$$

Two boundary conditions are required for each of these equations. The first three use the fact that the temperature of the ice and the water at the interface is the fusion temperature 
and the temperature at the bottom of the ice layer is zero:

$$
\left.T\right|_{\eta=0}=0,\left.\quad T\right|_{\eta=b}=\left.\chi\right|_{\eta=b}=1 .
$$

A heat flux condition is applied at the top of the water layer:

$$
\left.\frac{\partial \chi}{\partial \eta}\right|_{\eta=b+h}=E_{0 F}-\left.E_{1 F} \chi\right|_{b+h}
$$

where the two coefficients, $E_{0 F}$ and $E_{1 F}$, include kinetic energy, radiation, conduction, convection and evaporation. They may be time dependent if the air flow varies with time. The solution of equations (46) is then straightforward. The temperature profiles are:

$$
\begin{aligned}
T & =\frac{\eta}{b} \\
\chi & =1+\frac{E_{0 F}-E_{1 F}}{1+E_{1 F} h}(\eta-b) .
\end{aligned}
$$

The energy balance (45) can be expressed in its final form:

$$
S \frac{\partial b}{\partial t}=\frac{1}{b}-\frac{k_{f}}{k_{s}} \frac{E_{0 F}-E_{1 F}}{1+E_{1 F} h}
$$

The first term of the right hand side of equation (51) is proportional to $1 / b$. The potential singularity at $b=0$ is prevented by the restriction that the temperature of the solid substrate is constant and below the solidification temperature. This means that accretion always occurs initially and (51) only applies when $b \geq b_{f} \neq 0$. If a heat flux condition of the form (48) is applied at the substrate it is possible that $b_{f}=0$. However, in this situation the first term on the right hand side of (51) becomes $1 /\left(a_{0}+b\right)$, where $a_{0}>0$ is a constant and the potential singularity is eliminated. This situation is particularly relevant to ice accretion on aircraft in mild conditions.

The wet accretion problem is now in its final form. The accretion and fluid flow are governed by the coupled mass and energy balances, equations (32) and (51). The energy balance is relatively easy to deal with but the mass balance is highly nonlinear and difficult to solve. For this reason, the numerical solutions of $\S 4$ focus initially on the fluid flow. The coupled accretion and fluid flow problem is then a simple extension of the fluid flow results. 


\section{Reduction of the governing equations to standard forms}

During the initial stages all of the incoming fluid solidifies and the height $b$ is determined through the mass balance (36) or (37). The temperature is specified by equation (42). The accretion continues to be specified by (36) or (37) until the surface, $\eta=b$, reaches the phase change temperature. The accretion thickness at this stage is specified by equation (43). Subsequently fluid will appear. The flow depends on the geometry and this will be discussed in the following sections. The energy balance for wet accretion, (51), holds in all geometries. For simplicity the surface tension will be taken as constant in all the examples, so $\sigma \equiv 1$.

\subsection{Flat substrate}

When the substrate is flat the surface may be specified by setting $s_{1}=x, s_{2}=y$ and $\mathbf{R}=(x, y, 0)$. The first and second fundamental forms are

$$
\begin{array}{rlrl}
E & =\frac{\partial \mathbf{R}}{\partial x} \cdot \frac{\partial \mathbf{R}}{\partial x}=1 & G & =\frac{\partial \mathbf{R}}{\partial y} \cdot \frac{\partial \mathbf{R}}{\partial y}=1 \\
L & =\mathbf{n} \cdot \frac{\partial^{2} \mathbf{R}}{\partial x^{2}}=0 & N & =\mathbf{n} \cdot \frac{\partial^{2} \mathbf{R}}{\partial y^{2}}=0 .
\end{array}
$$

According to equation (11) the substrate and normal vectors are

$$
\mathbf{e}_{1}=(1,0,0) \quad \mathbf{e}_{2}=(0,1,0) \quad \mathbf{n}=(0,0,1)
$$

The fluid pressure, specified by equation (28), is

$$
p=p_{0}-C\left(\frac{\partial^{2}}{\partial x^{2}}(b+h)+\frac{\partial^{2}}{\partial y^{2}}(b+h)\right) .
$$

The flow is governed by equation (32) where the surface operator, equation (33), is

$$
\nabla_{s} \cdot \mathbf{Q}=\frac{\partial Q_{1}}{\partial x}+\frac{\partial Q_{2}}{\partial y}
$$

The fluxes are

$$
\begin{aligned}
Q_{1} & =-\left(\frac{\partial p}{\partial x}-B \mathbf{g} \cdot \mathbf{e}_{1}\right) \frac{h^{3}}{3}+A_{1} \frac{h^{2}}{2} \\
Q_{2} & =-\left(\frac{\partial p}{\partial y}-B \mathbf{g} \cdot \mathbf{e}_{2}\right) \frac{h^{3}}{3}+A_{2} \frac{h^{2}}{2}
\end{aligned}
$$


The pressure is defined by equation (52) and $\mathbf{g} \cdot \mathbf{e}_{1}=\cos \theta, \mathbf{g} \cdot \mathbf{e}_{2}=\cos \phi$ where $\theta$ and $\phi$ represent the inclination of the $x$ and $y$ axes to the horizontal.

In the absence of solidification $b \equiv 0$. If $\mathbf{W}=\mathbf{0}$ the standard equation for the flow of a thin film on a flat surface is retrieved. It has been studied extensively, see $[1,2]$ for example. With $\mathbf{W} \neq \mathbf{0}$ the model describes the flow of an evaporating or condensing thin film, see [2, $38]$ for example. With $b$ and $\mathbf{W}$ non-zero the model reduces to that describing ice accretion and water flow on a flat surface [4]. A version of this model is currently being used in the ICECREMO aircraft icing code $[11,22]$.

\subsection{Circular cylinder substrate}

In cylindrical polars the substrate may be parameterised with $s_{1}=R \theta, s_{2}=z$ (note, as discussed in $\S 2.1, s_{1}$ is a length). The substrate is then defined by $\mathbf{R}=(R \cos \theta, R \sin \theta, z)$, where $R$ is the constant cylinder radius. For flow on the outside of a cylinder the outward normal is $\mathbf{n}=(\cos \theta, \sin \theta, 0)$, flow on the inside requires $\mathbf{n}=-(\cos \theta, \sin \theta, 0)$. The first and second fundamental forms are therefore

$$
E=1 \quad G=1 \quad L= \pm \frac{1}{R} \quad N=0
$$

The curvature terms are

$$
\kappa_{1}=\frac{L}{E}= \pm \frac{1}{R} \quad \kappa_{2}=\frac{N}{G}=0
$$

where $\kappa_{1}>0$ denotes flow inside a cylinder, $\kappa_{1}<0$ denotes flow on the outside. The pressure is

$$
p=p_{0}-C^{\prime}\left[ \pm \frac{1}{R}+\epsilon \frac{b+h}{R^{2}}+\epsilon\left(\frac{1}{R^{2}} \frac{\partial^{2}}{\partial \theta^{2}}(b+h)+\frac{\partial^{2}}{\partial z^{2}}(b+h)\right)\right] .
$$

The flow is governed by equation (32) with

$$
\nabla_{s} \cdot \mathbf{Q}=\frac{1}{R} \frac{\partial Q_{1}}{\partial \theta}+\frac{\partial Q_{2}}{\partial z}
$$

The fluxes $Q_{1}$ and $Q_{2}$ are

$$
\begin{aligned}
Q_{1} & =-\left(\frac{1}{R} \frac{\partial p}{\partial \theta}-B \mathbf{g} \cdot \mathbf{e}_{\mathbf{1}}\right) \frac{h^{3}}{3}+A_{1} \frac{h^{2}}{2} \\
Q_{2} & =-\left(\frac{\partial p}{\partial z}-B \mathbf{g} \cdot \mathbf{e}_{\mathbf{2}}\right) \frac{h^{3}}{3}+A_{2} \frac{h^{2}}{2}
\end{aligned}
$$


where the pressure is defined by equation (58) and

$$
\mathbf{e}_{\mathbf{1}}=\frac{1}{E^{1 / 2}} \frac{\partial \mathbf{R}}{\partial s_{1}}=(-\sin \theta, \cos \theta, 0) \quad \mathbf{e}_{\mathbf{2}}=\frac{1}{G^{1 / 2}} \frac{\partial \mathbf{R}}{\partial s_{2}}=(0,0,1) \quad \mathbf{n}= \pm \mathbf{e}_{\mathbf{1}} \times \mathbf{e}_{\mathbf{2}} .
$$

Surface tension driven axisymmetric flow both inside and outside a cylinder has been considered in [2, 23 - 31]. Neglecting gravity, air shear, derivatives in the $\theta$ direction and setting $b=0, \mathbf{W}=\mathbf{0}$ the governing equation reduces to

$$
\frac{\partial h}{\partial t}+\frac{\partial}{\partial z}\left[C \frac{h^{3}}{3} \frac{\partial}{\partial z}\left(\frac{h}{R^{2}}+\frac{\partial^{2} h}{\partial z^{2}}\right)\right]=0 .
$$

This equation has been used to describe bubble motion in a capillary tube and the RayleighTaylor instability in a thin film $[23-25]$. The ratio $h / R^{2}$ is termed the hoop stress. It behaves like a negative gravity term, acting to pull the fluid away from the substrate and therefore destabilises the flow. With gravity acting in the $z$-direction another term proportional to $\partial h^{3} / \partial z$ is introduced into (63), this is discussed in [30,31]. Weidner et al [27] investigate flow on a horizontal cylinder. However, initially they retain the full curvature terms in order to permit droplet formation on the underside of the cylinder. This leads to the flux containing logarithmic terms, as discussed in $§ 2.2$. Subsequently these authors expand the $\log$ term with $h / R \ll 1$ to obtain a similar flux expression to that given above. Jensen [26] considers flow in a circular cylinder with small curvature along the axis. This equation is most easily retrieved by altering the above definition of the substrate to describe a torus $\mathbf{R}=R_{1}(\cos \theta, \sin \theta, 0)+R_{2}(\cos \theta \cos \phi, \sin \theta \cos \phi, \sin \phi)$ and setting $R_{2} \gg R_{1}$.

With $b$ and $\mathbf{W}$ non-zero the model describes solidification on the surface of a cylinder. This is of particular interest to the power transmission industry for icing on cables or icicle formation $[8,20]$.

\subsection{Non-circular cylinder substrate}

A non-circular cylinder may be described by the substrate $\mathbf{R}=(f(s), g(s), z)$. The parameter $s$ represents arc-length, so

$$
\left(\frac{\partial f}{\partial s}\right)^{2}+\left(\frac{\partial g}{\partial s}\right)^{2}=f_{s}^{2}+g_{s}^{2}=1
$$


where the subscript $s$ denotes derivatives with respect to that variable. The normal is $\mathbf{n}=$ $\pm\left(-g_{s}, f_{s}, 0\right)$, where the \pm sign determines whether the normal points inwards or outwards. The first and second fundamentals are

$$
E=1 \quad G=1 \quad L= \pm\left(-g_{s} f_{s s}+f_{s} g_{s s}\right)= \pm \frac{g_{s s}}{f_{s}} \quad N=0
$$

The curvatures are therefore

$$
\kappa_{1}= \pm \frac{g_{s s}}{f_{s}} \quad \kappa_{2}=0
$$

The fluid pressure is

$$
p=p_{0}-C^{\prime}\left[\kappa_{1}+\epsilon\left(h \kappa_{1}^{2}+\frac{\partial^{2} h}{\partial s^{2}}+\frac{\partial^{2} h}{\partial z^{2}}\right)\right] .
$$

The flow is governed by equation (32) with

$$
\nabla_{s} \cdot \mathbf{Q}=\frac{\partial Q_{1}}{\partial s}+\frac{\partial Q_{2}}{\partial z}
$$

where

$$
\begin{aligned}
& Q_{1}=-\left(\frac{\partial p}{\partial s}-G \mathbf{g} \cdot \mathbf{e}_{\mathbf{1}}\right) \frac{h^{3}}{3}+A_{1} \frac{h^{2}}{2} \\
& Q_{2}=-\left(\frac{\partial p}{\partial z}-G \mathbf{g} \cdot \mathbf{e}_{2}\right) \frac{h^{3}}{3}+A_{2} \frac{h^{2}}{2} .
\end{aligned}
$$

The pressure is specified by equation (65) and the surface vectors are specified by (11). The equations governing flow on a circular cylinder, derived in the previous section, may be retrieved by setting $s=R \theta$ and $f=\cos (s / R), g=\sin (s / R)$.

Schwartz \& Weidner [29] consider surface tension driven flow on an arbitrary twodimensional surface. Neglecting air shear, gravity, derivatives in the $z$-direction and setting $b=0, \mathbf{W}=\mathbf{0}$ equation (32), in this case, reduces to

$$
\frac{\partial h}{\partial t}+\frac{\partial}{\partial s}\left[C^{\prime} \frac{h^{3}}{3} \frac{\partial}{\partial s}\left(\kappa_{1}+\epsilon\left(\kappa_{1}^{2} h+\frac{\partial^{2} h}{\partial s^{2}}\right)\right)\right]=0,
$$

where $\kappa_{1}=g_{s s} / f_{s}$. There are two main differences between (67) and the corresponding equation in [29]. Firstly, equation (67) is in non-dimensional form. This makes it clear that 
when $\kappa_{1}=\mathcal{O}(1)$ and is non-constant it is the substrate shape alone that drives the flow to leading order. The problem in this case is governed by

$$
\frac{\partial h}{\partial t}+\frac{\partial}{\partial s}\left[C^{\prime} \frac{h^{3}}{3} \frac{\partial \kappa_{1}}{\partial s}\right]=0 .
$$

The second difference is that the term $\kappa_{1}^{2} h$ is absent in [29]. This occurs as a result of their expansion of the free surface curvature. Only two terms are taken in this expansion, $\kappa_{1}$ and $h_{s s}$. A neglected term, $\kappa_{1}^{2} h$, occurs at the same order as $h_{s s}$ and therefore should also be retained. This is particularly relevant when $\kappa_{1}= \pm 1 / R$ is constant. In this case $\kappa_{1}^{2} h=h / R^{2}$ is the hoop stress term observed in equation (63). When the curvature is small, so that $\kappa_{1}=\epsilon \kappa_{1}^{\prime}=\mathcal{O}(\epsilon)$ the flow is governed by

$$
\frac{\partial h}{\partial t}+\frac{\partial}{\partial s}\left[C \frac{h^{3}}{3} \frac{\partial}{\partial s}\left(\kappa_{1}^{\prime}+\frac{\partial^{2} h}{\partial s^{2}}\right)\right]=0
$$

This is the equation derived in [29], which is therefore strictly valid for non-constant curvatures of order $\epsilon$. Weidner et al [28] study an evaporating film with variable surface tension lying on an arbitrary two-dimensional surface. Their governing equation is obtained in the same manner as (69) with the inclusion of the shear term $A_{1} h^{2} / 2$, the mass loss $\mathbf{W} \cdot \mathbf{n}$ and $\sigma \neq 1$

\subsection{Spherical substrate}

In this case the surface is parametrised by $s_{1}=R \theta, s_{2}=R \phi$ where $\theta$ and $\phi$ represent the usual polar and azimuthal angles and $R$ is the radius of the sphere. The substrate is then $\mathbf{R}=R(\sin \theta \cos \phi, \sin \theta \sin \phi, \cos \theta)$ and the normal $\mathbf{n}= \pm \mathbf{R} / R$. The first and second fundamental forms are

$$
E=1 \quad G=\sin ^{2} \theta \quad L=\mp \frac{1}{R} \quad N=\mp \frac{1}{R} \sin ^{2} \theta .
$$

The curvatures are therefore

$$
\kappa_{1}=\mp \frac{1}{R}=\kappa_{2}
$$

The fluid pressure is

$$
p=p_{0}-C^{\prime}\left(\mp \frac{2}{R}+\epsilon\left[\frac{2}{R^{2}}(b+h)+\frac{1}{R^{2}} \frac{\partial^{2}}{\partial \theta^{2}}(b+h)+\frac{1}{R^{2} \sin ^{2} \theta} \frac{\partial^{2}}{\partial \phi^{2}}(b+h)\right]\right) .
$$


The flow is governed by (32) with

$$
\nabla_{s} \cdot \mathbf{Q}=\frac{1}{R} \frac{\partial Q_{1}}{\partial \theta}+\frac{1}{R \sin \theta} \frac{\partial Q_{2}}{\partial \phi}
$$

and

$$
\begin{aligned}
Q_{1} & =-\left(\frac{1}{R} \frac{\partial p}{\partial \theta}-B \mathbf{g} \cdot \mathbf{e}_{\mathbf{1}}\right) \frac{h^{3}}{3}+A_{1} \frac{h^{2}}{2} \\
Q_{2} & =-\left(\frac{1}{R \sin \theta} \frac{\partial p}{\partial \phi}-B \mathbf{g} \cdot \mathbf{e}_{2}\right) \frac{h^{3}}{3}+A_{2} \frac{h^{2}}{2}
\end{aligned}
$$

\section{Numerical solution method}

The general mathematical model for fluid flow and accretion is specified by equations (32) and (51). In the absence of accretion the flow model is typical for thin film free surface flows which are notoriously difficult to solve both analytically and numerically. If the fourthorder surface tension term is neglected then shocks are likely to develop, particularly in the vicinity of a moving contact line. Another difficulty associated with the contact line is the inability of the lubrication approximation to accurately predict fluid behaviour in this region [32]. It is well-known that the no-slip velocity boundary condition at $z=0$ leads to a multi-valued velocity field in the vicinity of the contact line. This results in a non-integrable stress singularity. A number of methods to overcome this difficulty have been developed, such as the introduction of a precursor layer, replacing the no-slip condition by a Navier slip condition or allowing rolling in an inner region and matching to the outer lubrication model [1]. In the following a precursor layer of thickness $h_{p}$ will be employed. Since the fluid flow is the most problematic aspect of the numerical solution this will be dealt with first in $§ 4.1$ and 4.2. The coupling with the accretion model is a relatively simple extension and will be dealt with in $\S 4.3$.

\subsection{Fluid flow on a two-dimensional surface}

In the absence of accretion, the equation governing the two-dimensional fluid flow is:

$$
\frac{\partial h}{\partial t}+\frac{\partial Q_{1}}{\partial s_{1}}=-\mathbf{W} \cdot \mathbf{n},
$$


where $Q_{1}$ is specified by equation (34). This is discretised with constant space and time steps $\Delta s_{1}$ and $\Delta t$. The water height at the centre of the $i-t h$ cell of the grid at time $t=k \Delta t$ is denoted $h_{i}^{k}$. Provided the numerical domain is sufficiently large, equation (76) may be solved with the boundary conditions that the first and second derivatives of the flux are zero (due to the presence of the precursor layer the flux is not necessarily zero at the boundaries).

A typical finite difference scheme in conservative form for equation $(76)$ is $[33,35]$ :

$$
h_{i}^{k+1}=h_{i}^{k}-\mathbf{W} \cdot \mathbf{n} \Delta t-\frac{\Delta t}{\Delta s}\left(Q_{1_{i+1 / 2}}-Q_{1_{i-1 / 2}}\right),
$$

where $Q_{1_{i+1 / 2}}$ denotes the flux at the boundary of the $i-t h$ and $(i+1)-t h$ cells. It depends on the water heights around the $i-t h$ cell, $h_{i-3}$ to $h_{i+3}$, calculated at time $k \Delta t$ and $(k+1) \Delta t$. Over most of the domain, the dominant terms in the flux are the shear stress, $A_{1} h^{2} / 2$, and the gravity term, $B \mathbf{g} \cdot \mathbf{e}_{\mathbf{1}} h^{3} / 3$. If all other terms in the flux are neglected, equation (76) is likely to develop a shock at the moving front. Preventing the eventual shock from developing is the surface tension term, which increases with the curvature. However, in many practical situations there will exist a region of sufficiently high curvature at the front of the flow to cause numerical problems. To prevent this a shock capturing technique, as described in [4], will be employed.

Since equation (76) is nonlinear, calculating all terms implicitly in the flux is not possible. An alternative method is presented in [36] where the derivatives of the film height are evaluated at time $t=(k+1 / 2) \Delta t$, using the Crank-Nicolson method, all other terms are calculated explicitly, at time $t=k \Delta t$. To achieve this the flux is divided into two parts, $Q_{1}=Q^{I}+Q^{I I}$, where:

$$
\begin{aligned}
Q^{I} & =\frac{h^{3}}{3} \frac{C}{E^{1 / 2}}\left(\kappa_{1}^{2} \frac{\partial h}{\partial s_{1}}+\frac{1}{E} \frac{\partial^{3} h}{\partial s_{1}^{3}}\right) \\
Q^{I I} & =\frac{h^{3}}{3}\left(\frac{C^{\prime}}{E^{1 / 2}}\left[\frac{\partial \kappa_{1}}{\partial s_{1}}+\epsilon h \frac{\partial \kappa_{1}^{2}}{\partial s_{1}}\right]-\frac{1}{E^{1 / 2}} \frac{\partial p_{0}}{\partial s_{1}}+B \mathbf{g} \cdot \mathbf{e}_{\mathbf{1}}\right)+A_{1} \frac{h^{2}}{2}
\end{aligned}
$$

The first term is discretised using a Crank-Nicolson scheme, see [36]:

$$
Q_{i+1 / 2}^{I}=\frac{\left(h_{i+1 / 2}^{k}\right)^{3}}{3} \frac{C}{E^{1 / 2}}\left\{\left(\left.\frac{1}{2} \frac{\partial^{3} h}{\partial s^{3}}\right|_{i+1 / 2} ^{k+1}+\left.\frac{1}{2} \frac{\partial^{3} h}{\partial s^{3}}\right|_{i+1 / 2} ^{k}\right)\right.
$$




$$
\left.+\kappa_{1}^{2}\left(\left.\frac{1}{2} \frac{\partial h}{\partial s}\right|_{i+1 / 2} ^{k+1}+\left.\frac{1}{2} \frac{\partial h}{\partial s}\right|_{i+1 / 2} ^{k}\right)\right\} .
$$

The second part, $Q^{I I}$, contains the terms likely to produce shocks. Numerical experiments carried out in $[4,44]$ indicate that the Roe \& Sweby scheme with the 'Superbee' limiter provides accurate solutions whilst permitting relatively large time-steps. This requires that this part of the flux is split once again:

$$
\begin{aligned}
Q_{i+1 / 2}^{I I} & =\left(1-c_{i+1 / 2}\right) Q_{i+1 / 2}^{U P}+c_{i+1 / 2} Q_{i+1 / 2}^{L W}, \\
c_{i+1 / 2} & =\max (0, \min (2 r, 1), \min (r, 2)), \\
r & =\frac{\left(\left|a_{i+1 / 2-\mathrm{a} \mid}\right|-\Delta t a_{i+1 / 2-\mathrm{a}}^{2} / \Delta s_{1}\right)\left(h_{i+1-\mathrm{a}}-h_{i-\mathrm{a}}\right)}{\left(\left|a_{i+1 / 2}\right|-\Delta t a_{i+1 / 2}^{2} / \Delta s_{1}\right)\left(h_{i+1}-h_{i}\right)}, \\
\mathrm{a} & =\operatorname{sign}\left(a_{i+1 / 2}\right)
\end{aligned}
$$

where $a_{i+1 / 2}$ denotes the wave speed, $Q^{U P}$ and $Q^{L W}$ represent the upwind and Lax-Wendroff schemes:

$$
\begin{aligned}
a_{i+1 / 2} & = \begin{cases}\left(Q_{i+1}^{I I}-Q_{i}^{I I}\right) /\left(h_{i+1}-h_{i}\right) & \text { if } h_{i+1}-h_{i} \neq 0, \\
\partial Q^{I I} /\left.\partial h\right|_{i+i / 2} & \text { if } h_{i+1}-h_{i}=0,\end{cases} \\
Q_{i+1 / 2}^{U P} & =\frac{1}{2}\left(Q_{i}^{I I}+Q_{i+1}^{I I}\right)-\frac{1}{2} \operatorname{sign}\left(a_{i+1 / 2}\right)\left(Q_{i+1}^{I I}-Q_{i}^{I I}\right), \\
Q_{i+1 / 2}^{L W} & =\frac{1}{2}\left(Q_{i}^{I I}+Q_{i+1}^{I I}\right)-\frac{\Delta t}{2 \Delta s} a_{i+1 / 2}^{2}\left(h_{i+1 / 2}-h_{i-1 / 2}\right),
\end{aligned}
$$

and $Q_{i}^{I I}$ denotes the second part of the flux calculated at the centre of the $i-t h$ cell with a centred scheme at time $k \Delta t$. Equation (76) is now fully discretised and the film height $\left(h_{i}^{k+1}\right)_{i=1 . . n}$ may be determined by solving equation (77) with the flux specified by equations (78) and (79). This leads to a system of $n$ linear equations and the problem reduces to the inversion of a pentadiagonal matrix. For full details see [36, 44]

In Figure 2 a typical result is shown for flow on a horizontal surface. The rate at which fluid impacts on the surface is

$$
\mathbf{W} \cdot \mathbf{n}=-0.5 \exp \left(-3.16 s_{1}^{2}\right)
$$

This form is chosen to match physically realistic examples, such as the one discussed in $\S 4.5$. The flow is driven primarily by air shear. The height-scale is chosen as the equilibrium height 
on a horizontal surface, hence $h=1$ over most of the domain. The velocity scale, $U$, is chosen to make $A_{1}=1$. The various curves represent the film profile at $t=1,3,4,4.5,5,7,9,11$, 15. Since the flux $Q_{1}$ involves terms proportional to $h^{3}$ and $h^{2}$, when $h \ll 1$ the film profile is determined by the balance between the time derivative and the incoming fluid in equation (76). The early time profile is therefore approximately proportional to the Gaussian shape specified by (80). The precursor film $h=h_{p}=0.05$, can clearly be seen on either side of the Gausssian. As $h$ increases the air shear term enters the dominant balance and the fluid is driven to the right. A capillary ridge begins to develop after $t=2.5$ and the height of this ridge increases until around $t=10$ after which the height remains constant. The shape of the capillary ridge may be determined approximately using the method described in $[4,36,37]$. After $t=5$ the film profile behind the ridge is determined by the balance between air shear and the incoming fluid. In this case the bulk film shape may be determined analytically

$$
h=\sqrt{h_{p}^{2}+0.5 \sqrt{\frac{\pi}{3.16}}\left[1+\operatorname{erf}\left(\sqrt{3.16} s_{1}\right)\right]} .
$$

Matching the bulk and ridge solutions then provides a profile against which the numerical solution may be verified. However, since the results are too close to distinguish, only the numerical solution is shown in Figure 2. Further details may be found in [4]. The numerical solution has also been checked against the explicit solution method described in [4]. The curves match almost exactly, however the current calculation took approximately thirty seconds (fifty times less than the explicit solution).

\section{Figure 2 near here}

When the surface is inclined, with gravity acting against the shear stress, the evolution of the water layer is very different. Figure 3 shows the film profile for flow on a surface inclined at $20^{\circ}$ to the horizontal, again with a shear stress $A_{1}=1$ and $B \mathbf{g} \cdot \mathbf{e}_{1}=-0.857$, at times $t=$ $1,3,4,4.5,5,7,9,11,15$. The example has been chosen so that gravity almost balances the shear. At early times the fluid height reflects the incoming Gaussian profile. As the height increases motion to the right occurs, due to the shear stress, and to the left, due to gravity. The fluid on the right initially forms a capillary ridge which grows in height, until a critical 
value around $h=1.2$, when it ceases to grow and starts to spread out. The fluid continues to flow in this manner, with no discernible leading capillary ridge. This phenomenon has been observed in surface tension gradient driven flows [41]. At the left-hand edge of this flat region the fluid dips. Moving further to the left the height increases to the gravity dominated region which behaves in a more usual manner, with a capillary ridge forming at early times. The ridge increases in height, to a certain value, and a flat bulk flow region begins to form behind the ridge at later times.

Figure 3 near here

\subsection{Fluid flow on a three-dimensional surface}

In the absence of accretion, the equation governing the three-dimensional fluid flow is (32) with $b \equiv 0$. In this case the solution space is divided into an $m \times n$ grid, whose cell sizes are denoted $\Delta s_{1}$ and $\Delta s_{2}$. The film height in the centre of cell $(i, j)$ at time $t=k \Delta t$ is denoted $h_{i, j}^{k}$.

The generalisation of the two-dimensional scheme to three dimensions is not straightforward. In three dimensions, solving for the film height requires the inversion of a broadly banded matrix. An alternative method is to replace the implicit scheme by an Alternating Direction Implicit (ADI) scheme, or Peaceman-Rachford non-homogeneous scheme [34, 39, 40]. The time step is then divided into two equal parts: when $t \in[k, k+1 / 2] \Delta t$ the flux is evaluated implicitly in the $s_{1}$ direction and explicitly in the $s_{2}$ direction; during $t \in[k+1 / 2, k+1] \Delta t$ the flux is evaluated explicitly in the $s_{1}$ direction and implicitly in the $s_{2}$ direction. The pressure gradient involves two cross derivatives $\partial^{3} h /\left(\partial s_{1}^{2} \partial s_{2}\right)$ and $\partial^{3} h /\left(\partial s_{1} \partial s_{2}^{2}\right)$. These could be calculated implicitly but they would add non-pentadiagonal terms to the matrix. If the cross terms are evaluated explicitly then the film height may be determined by inverting two pentadiagonal matrices, see [27, 38]. Again all the explicit terms are evaluated using the Roe and Sweby scheme with the Superbee limiter.

Figure 4 near here 
In Figure 4 an example of flow on an inclined flat surface is shown. The slope is such that $\hat{\mathrm{g}} \cdot \mathbf{e}_{1}=\hat{\mathrm{g}} \cdot \mathbf{e}_{2}=-0.4$. The surface shear components act against gravity with $A_{1}=A_{2}=1$. The height is scaled with $H=0.1286 \mathrm{~mm}$ (the equilibrium height for the equivalent twodimensional problem), the length is scaled with $L=8.3 \mathrm{~cm}$. The incoming fluid is described by

$$
\mathbf{W} \cdot \mathbf{n}=-0.5 \exp \left(-3.16\left(s_{1}^{2}+s_{2}^{2}\right)\right)
$$

The space and time-steps employed in the calculation were $\Delta s_{1}=\Delta s_{2}=0.0015, \Delta t=0.0005$ and the figure shows the result at $t=15$. Shear and gravity forces are mainly acting along the diagonal joining the left and the right corners.

This example includes the different patterns likely to develop for this type of flow:

- The left extremity of the curve is mainly driven by gravity, see the enlargement in 5 (a). Two waves appear on the side of the flow due to the gravity driven spreading perpendicular to the main flow direction. These two waves join and form a single capillary ridge which ends at a high peak. This shape strongly resembles the two dimensional curves described in the previous section.

- The right extremity of the flow shows a very different pattern, as may be observed from the enlargement in Figure 5 (b). The front forms a semi-circular flat plateau, surrounded by small side waves created by the balance between gravity driven spreading and surface tension. There is a distinct step, $\Delta h \approx 0.2$ from the bulk height to the plateau height.

- Between the two fronts, the bulk region consists of an approximately flat section which then slowly increases in height away from the right. At the left extremity the bulk region levels off again before the peak is reached. The sides of this region display a small capillary ridge due to the sideways flow driven by gravitational spreading. The two, approximately constant heights on either side of the origin are determined by the balance between gravity and shear stress. The two heights are different because near the origin, where most fluid impacts, the film is sufficiently thick for gravity to dominate 
and drive the most of the fluid to the left. Mathematically, the two different heights explain the two different type of fronts. The left hand region has a slightly increasing height and the flux $Q^{I I}$ is convex, so a Lax shock appears here. On the other side, the water height is decreasing and $Q^{I I}$ is concave, consequently an undercompressive shock develops at the right hand front [42, 43].

Figure 5 near here

\subsection{Coupled flow and accretion}

As mentioned before, there is an initial period when only dry accretion occurs. In which case the thickness is determined by equation (36). The discretised form is

$$
b_{i, j}^{k+1}=b_{i, j}^{k}-\mathbf{W} \cdot \mathbf{n} \Delta t .
$$

Fluid appears when a point or area of the top surface reaches the fusion temperature. The accretion profile at this time is specified by equation (43). When fluid appears, equation (83) may still hold in certain regions where the fluid has not yet flowed. Over the rest of the domain the accretion is governed by equation (51) which is coupled to the flow equation (32). In discrete form these are

$$
\begin{array}{r}
b_{i, j}^{k+1}=b_{i, j}^{k}+\frac{1}{S}\left(\frac{1}{b_{i, j}^{k}}-\frac{k_{f}}{k_{s}} \frac{E_{0 F}-E_{1 F}}{1+E_{1 F} h_{i, j}^{k}}\right) \Delta t \\
h_{i, j}^{k+1}=h_{i, j}^{k}-\frac{\Delta t}{\Delta s_{1}}\left(Q_{1_{i+1 / 2, j}}-Q_{1_{i-1 / 2, j}}\right)-\frac{\Delta t}{\Delta s_{2}}\left(Q_{2_{i, j+1 / 2}}-Q_{2_{i, j-1 / 2}}\right) \\
\quad-\frac{1}{S}\left(\frac{1}{b_{i, j}^{k}}-\frac{k_{f}}{k_{s}} \frac{E_{0 F}-E_{1 F}}{1+E_{1 F} h_{i, j}^{k}}\right) \Delta t-\mathbf{W} \cdot \mathbf{n} \Delta t .
\end{array}
$$

However, the extent of the wet domain is not known a priori. In order to determine which model, dry or wet accretion, is appropriate at a given point, the following method is used:

- The fluid and accretion heights are calculated using equations (84) and (85). These require that $b_{i, j}^{k} \neq 0$. For this reason, a precursor ice film $b_{p}$ is specified (typically this is taken the same as the fluid precursor, $\left.b_{p}=0.05\right)$.

- If the new calculated film height is greater than the precursor film, $h_{i, j}^{k}>h_{p}$, the wet accretion model is taken as correct. 
- If the new calculated fluid height is smaller than the precursor film, $h_{i, j}^{k}<h_{p}$, this means that the accretion is dry. The calculated value for the film height is replaced by the precursor film, $h_{p}$, and the new ice height is calculated using equation (83).

As the model stands, mass is not conserved. The problem occurs at the interface between wet and dry accretion. At the dry side of the accretion, the flux takes a small constant value due to the presence of the precursor film. At the wet side, the flux depends on the height from the neighbouring points and therefore the wet and dry flux values are not necessarily the same on either side of the interface. To correct this deficiency, in dry regions, equation (83) is modified to:

$$
b_{i, j}^{k+1}=b_{i, j}^{k}-\mathbf{W} \cdot \mathbf{n} \Delta t-\frac{\rho_{f}}{\rho_{s}}\left(\frac{Q_{1_{i+1 / 2, j}}-Q_{1_{i-1 / 2, j}}}{\Delta s_{1}}+\frac{Q_{2_{i, j+1 / 2}}-Q_{2_{i, j-1 / 2}}}{\Delta s_{2}}\right) \Delta t .
$$

Since the flux is constant in the precursor film, the new term on the right hand side is only non-zero at the dry/wet interface. This method ensures continuity and physically sensible results for the film height as well as mass conservation.

The numerical solution for flow on an accreting surface is therefore determined by first calculating the accretion profile immediately before fluid appears, $b=b_{f}$. Subsequently equations (84) and (85) hold over the wet region and equation (86) holds in the dry regions.

Figure 6 shows (a) the accretion and (b) the fluid film on a flat plane at $t=60$ with the same conditions to that of $\S 4.2$. The energy terms are given by

$$
\begin{aligned}
& E_{0 F}=0.235-0.0966 \mathbf{W} \cdot \mathbf{n}, \\
& E_{1 F}=0.164-0.095 \mathbf{W} \cdot \mathbf{n} .
\end{aligned}
$$

These correspond to a physical situation where water droplets exist in a flow field at temperature $272 \mathrm{~K}$, the substrate temperature is $271 \mathrm{~K}$, the heat transfer coefficient is $500 \mathrm{~W} / \mathrm{K} / \mathrm{m}^{2}$, the free stream velocity is $\mathbf{V}_{\infty}=(100,0,0) \mathrm{m} / \mathrm{s}$ and the incoming fluid is described by equation (82). The temperatures are chosen close to fusion temperature so that a significant water layer may develop. Taking the height and velocity scales of $\S 4.1$ gives the following non-dimensional numbers for the problem

$\epsilon=1.55 \times 10^{-3}, \quad A_{1}=1, \quad B=2.52, \quad C^{\prime}=2.72 \times 10^{-3}, \quad S=0.824$. 
Figure 6 near here

The accretion shown in Figure 6 (a) shows a central Gaussian region, corresponding to the incoming fluid. If the fluid were to solidify immediately upon impact then the accretion would have a Gaussian profile with a maximum height $b=-\left.\rho_{f} \mathbf{W} \cdot \mathbf{n}\right|_{s_{1}=s_{2}=0} t / \rho_{s} \approx 33.3$ (where $\rho_{s}=900, \rho_{f}=1000 \mathrm{~kg} / \mathrm{m}^{3}$ ). For the present situation fluid flow has altered the Gaussian. On the right the hump is caused by the solidification of the air shear driven flow, on the left a thin hump occurs due to the gravity driven flow. The different amounts of accretion on either side of the centre reflect the magnitude of the driving force and ease with which the fluid moves over a dry surface. Clearly surface shear is the dominant driving force, even though the Bond number $B>A$ the surface shear. This is due to the fact that $A$ multiplies $h^{2}$ whilst $B$ multiplies $h^{3}$ and the water height remains low for a significant length of time.

It is clear from Figure 6 (b) that the flow with accretion is qualitatively different to that without (shown in Figure 4). A shock still forms on the left of the picture, on the right side of the figure the flow is driven to the right by shear stress, gravity also acts to drive the flow to the left and at the same time accretion removes fluid. This combination results in the film height decreasing monotonically to the precursor layer and no shock or flat region appears on the right side.

\subsection{Flow and accretion on a sphere}

Accretion on a sphere is now studied for the case of a gravity driven film. The two coordinates $s_{1}$ and $s_{2}$ are defined as the lengths along the parallels and meridians respectively. However, for clarity, the results will be shown using the two spherical coordinate angles $\phi$ and $\theta$. Gravity acts in the direction of increasing $\theta$.

The simulation is carried out with the following parameters:

$$
\begin{aligned}
& B=1, \quad C^{\prime}=6.42 \times 10^{-5}, \quad S=0.43, \\
& E_{0 F}=0.00144-0.0207 \mathbf{W} \cdot \mathbf{n}, \quad E_{1 F}=0.00307-0.04439 \mathbf{W} \cdot \mathbf{n}, \quad \epsilon=3.53 \times 10^{-4}, \\
& A_{1}=0, \quad A_{2}=0, \quad \mathbf{W} \cdot \mathbf{n}=-0.7 \exp \left(-13.87 s^{2}\right),
\end{aligned}
$$


where $s=\sqrt{s_{1}^{2}+s_{2}^{2}}$ denotes the distance to the origin along the surface. These values correspond to far field and substrate temperatures $T_{\infty}=272 \mathrm{~K}$ and $T_{s}=271 \mathrm{~K}$, the far field velocity is $\mathbf{V}_{\infty}=(5,0,0) \mathrm{m} \cdot \mathrm{s}^{-1}$ and the heat transfer coefficient is assumed constant, 5 $\mathrm{W} / \mathrm{m}^{2} / K$. The scales are $H=0.12 \mathrm{~mm}, L=33.98 \mathrm{~cm}, \tau=2.40 \mathrm{~s}$. The height-scale is the appropriate choice for a gravity driven flow on a vertical surface.

Figures 7 (a), (b) near here

Under these conditions water appears on the surface at $t=4.28$. The ice accretion at $t=30$ (corresponding to approximately 72s) is shown in Figure 7(a). If all of the incoming fluid had frozen then the ice shape would be proportional to the Gaussian with maximum height $b=23.3$. The flattening over the top region indicates that water has been present and moved away from the central region. The film height is shown in Figure 7(b). The liquid is clearly pushed to the right of the picture (increasing $\theta$ ) by gravity. As with the flat inclined surface example, a shock develops at the moving front. However, the wave here is much smaller due to the lower water level. The simulation has been stopped at this time to avoid the problem which would occur as fluid accumulates at the bottom of the sphere.

\subsection{Flow and accretion on an aerofoil}

A more practical application of the current theory will now be described, that of ice accretion on an aerofoil. This is particularly relevant to ice growth on aircraft and wind turbines, see $[8,11,45]$ for example.

A good approximation to the top half of a NACA0012 aerofoil (with constant crosssection) is $\mathbf{R}=(x(p), y(p), z)$ where

$$
\begin{aligned}
& x(p)=\sum_{i=0}^{8} x_{i} p^{i}(1-p)^{(8-i)} \\
& y(p)=\sum_{i=0}^{8} y_{i} p^{i}(1-p)^{(8-i)}
\end{aligned}
$$

and $p \in[0,1]$. The points $\left(x_{i}, y_{i}\right)$ are

$$
\begin{aligned}
& (0.0,0.0),(0.0,1.48448),(0.99988,9.76164),(5.99984,27.39464),(15.00003,40.74980), \\
& (19.99984,31.20544),(14.99988,12.59776),(6.00000,2.42248),(1.00000,0.01050),
\end{aligned}
$$


see [46]. The bottom half of the aerofoil is obtained by symmetry. The surface therefore forms part of a non-circular cylinder, as discussed in $\S 3.3$ (however $p$ does not represent arc-length, so equation (64) does not hold). The arc-length co-ordinate $s$ is, chosen so that $s=0$ at the leading edge, $s<0$ is the bottom half of the aerofoil and $s>0$ the top half.

The model inputs were determined using FLUENT V [47] over a clean aerofoil. The temperatures are the same as in the previous examples, i.e. $T_{\infty}=272, T_{s}=271 \mathrm{~K}$. The collection efficiency may be defined as the ratio between the mass of impinging droplets to the incoming mass that would hit the substrate if the trajectories were straight lines [48]. The collection efficiency must therefore be less than 1. For the current problem a series of droplets of radius $r=50 \mu \mathrm{m}$ was introduced into the FLUENT flow solution and the air had a liquid water content $\rho_{a}=0.001 \mathrm{~kg} / \mathrm{m}^{3}$. The droplet impact points were recorded to provide the collection efficiency shown in Figure 6 (a). The droplets in the air flow are relatively small and tend to follow the air flow. The efficiency is therefore a maximum near the leading edge of the aerofoil where the air flow turns sharply and the droplets cannot follow. On either side the efficiency decreases rapidly as less droplets impact and more follow the airflow. Beyond the limits $|s|=1$ all the droplets flow around the wing. Ideally the collection efficiency should be a smooth curve. The oscillations in the figure indicate that a relatively small number of droplets have impacted in that region so leading to inaccuracies in the calculation. The flow solution obtained by FLUENT provides the shear stress, pressure and heat transfer coefficient shown in Figures 6 (b), (c), (d). These are all shown in dimensional form. The shear stress is negative for $s<0$, since the air flow is in the negative $s$ direction on the bottom surface. The magnitude of the shear stress is greatest just before and after the leading edge, with a maximum value 15.5Pa. For $s>0$ the stress is positive since the air flows in the positive $s$ direction. The ambient pressure is 101300Pa. Near the leading edge the pressure is greater than this value. For $|s|>0.3$ the pressure is less than this value. The symmetric form for the aerofoil shape, given by equation (88), means that both sides have equal suction and no lift would be generated. In reality an aerofoil requires asymmetry to generate lift in horizontal flight. The heat transfer coefficient is also highest at the leading 
edge. The peak corresponds to a dimensional value of approximately $545 \mathrm{~W} / \mathrm{m}^{2} \mathrm{~K}$. Figures 7 (a), (b) show the ice and water thicknesses on the aerofoil at times $t=500,1000,1500$ (corresponding to 3, 6, 9 minutes). Initially the ice shape is proportional to the collection efficiency, so there is a high peak in the centre and no ice beyond $|s|=1$. Since the ambient conditions are mild, water appears at an early time, $t=7$, and starts to produce ice away from this region. When $t=500$ the ice extends to $|s| \approx 1.3$, at $t=1500$ the ice reaches $|s| \approx 2.2$. The ice shape is relatively difficult to interpret due to the competing effects. However, it is likely that a peak occurs at the centre because the water impacting in this region is removed very slowly. Near the stagnation point the shear stress is low and the only driving force is gravity. The thin water film therefore moves very slowly and provides a large source for new ice. Away from this region the air shear drives the fluid outwards to extend the limits of the ice region.

The water film, shown in Figure 7 (b) has two peaks around $|s|=0.7$. These are caused by the water (except in the immediate vicinity of $s=0$ ) being driven away from the centre. As the fluid moves to the thin ice region it starts to freeze and so the water height decreases rapidly near the accretion edge.

\section{Conclusion}

A model for the flow of a thin, accreting film has been developed. In the absence of solidification, the model for fluid flow is the first fully three-dimensional thin film formulation on an arbitrary surface described in the literature. As such it can be applied to a wide variety of physically realistic thin film free surface flows. The various limiting cases of this model have been shown to capture previous systems on flat and curved surfaces in both two and three dimensions and also clarified the conditions under which these models are valid. When solidification is included the model can be reduced to a previous one concerning ice accretion on a flat surface.

There are a vast number of papers dealing with thin film free surface flows. These concern not only the different applications and flow regimes but also the mathematical properties 
of the governing equations. The addition of solidification to the problem adds a whole new level of complexity and interest to the problem. The numerical solutions show that the flow characteristics change considerably even when the accretion rate is small.

The model has a number of limitations. For example, the rate at which fluid enters the system must be sufficiently slow for the temperature to equilibrate to an approximately linear profile. The thermal mass of the substrate must be significantly larger than that of the accretion. The fluid is Newtonian, most coatings are non-Newtonian. A number of these restrictions are relatively simple to relax. For example, it is a simple matter to allow heat transfer between the substrate and the accretion. This will permit the modelling of antiicing systems and icing of cables, where the ice accretion may be significantly larger than the cable. In the case of a non-solidifying flow there are a variety of other possible driving forces which have not been included in this work. However, removing the restrictions and investigating different driving forces will be the subject of future research.

Verification of the model with accretion is difficult. The most easily available source of data comes from ice accretion studies. On aircraft the conditions are very severe and inaccuracies will come from the experimental observations, the flow solution obtained via FLUENT and the accretion model itself. With atmospheric icing the ambient conditions can change throughout the course of an icing event and knowing the precise inputs for the model is problematic. However, it is hoped in the future to verify this model through controlled experiments for ice accretion on cables. The model for accretion on a flat surface is already being used and tested in an aircraft icing code and results from this study will be published.

\section{Acknowledgement}

TM would like to thank the Oxford Centre for Industrial and Applied Mathematics (OCIAM) at the University of Oxford for providing the facilities where the majority of this work was written up and for appointing him as a Visiting Research Fellow. 


\section{Nomenclature}

\begin{tabular}{|c|c|}
\hline$A_{i}$ & Surface shear \\
\hline$B$ & Bond number \\
\hline$b$ & Ice thickness \\
\hline$C$ & Inverse capillary number \\
\hline$C^{\prime}$ & Non-standard inverse capillary number $C=\epsilon C^{\prime}$ \\
\hline$E, F, G$ & First fundamental forms \\
\hline$E_{i D}$ & Energy term in dry accretion \\
\hline$E_{i F}$ & Energy term in wet accretion \\
\hline $\mathbf{e}_{1}, \mathbf{e}_{2}$ & Unit substrate vectors \\
\hline$H$ & Height scale \\
\hline$k_{f}, k_{s}$ & Thermal conductivity of fluid and solid \\
\hline$L, M, N$ & Second fundamental forms \\
\hline$L$ & Length-scale \\
\hline$L_{f}$ & Latent heat of fusion \\
\hline $\mathbf{n}$ & Unit vector normal to substrate \\
\hline $\mathrm{Pe}$ & Peclet number \\
\hline$p$ & Fluid pressure \\
\hline $\mathbf{Q}=\left(Q_{1}, Q_{2}\right)$ & Fluid volume flux vector \\
\hline $\mathbf{R}$ & Function defining the substrate \\
\hline $\mathbf{r}$ & Function defining current point \\
\hline$S$ & Stefan number \\
\hline$s_{i}$ & Surface co-ordinates \\
\hline$T$ & Temperature in solid \\
\hline$T_{f}$ & Fusion temperature \\
\hline$T_{s}$ & Substrate temperature \\
\hline$T_{\infty}$ & Far field temperature \\
\hline $\mathbf{u}=(u, v, w)$ & Fluid velocity vector \\
\hline
\end{tabular}




$\begin{array}{ll}\mathbf{V}_{\infty} & \text { Velocity vector for far field air flow } \\ \mathbf{W} & \text { Local velocity of fluid droplets } \\ W & \text { Maximum value of } \mathbf{W} \\ \epsilon & \text { Small parameter, } \epsilon=H / L \\ \kappa_{1}, \kappa_{2} & \text { Curvature in }\left(s_{1}, s_{2}\right) \text { directions } \\ \eta & \text { Co-ordinate perpendicular to substrate } \\ \rho & \text { Density } \\ \chi & \text { Temperature in fluid } \\ \tau & \text { Time-scale }\end{array}$

\section{References}

1. Myers T.G. Thin films with high surface tension. SIAM Review 40(3), pp441-462 1998.

2. Oron A., Davis S.H. \& Bankoff S.G. Long-scale evolution of thin liquid films. Rev. Modern Phys. 69(3) pp931-980 1997.

3. Poots G.I. Ice and snow accretion on structures, Research Studies Press, 1996.

4. T.G. Myers, J.P.F. Charpin \& C.P. Thompson Slowly accreting ice due to supercooled water impacting on a cold surface. Submitted to Phys. Fluids, July 2000.

5. Stefan J. Über einige Probleme der Theorie der Wärmeleitung. S.-B. Wien. Akad. Mat. Natur. 98 pp473-484 1889.

6. Stefan J. Über die Theorie der Eisbildung, insbesondere über die Eisbildung im Polarmeere. S.-B. Wien. Akad. Mat. Natur. 98 pp965-983 1889.

7. Lock G.S.H. The growth and decay of ice. Cambridge University Press (1990).

8. L. Makkonen "Models for the growth of rime, glaze icicles and wet snow on structures" Phil. Trans. R. Soc. Lond. A 3582913 (2000). 
9. M. Farzaneh "Ice accretions on high-voltage conductors and insulators and related phenomena" Phil. Trans. R. Soc. Lond. A 3582971 (2000).

10. P. Sparaco Swedish crash prompts clear-ice guidelines, Aviation Week and Space Technology, 10 pp49-50, 1994.

11. R.W. Gent, N.P. Dart \& J.T. Cansdale Aircraft icing, Phil. Trans. R. Soc. Lond. A $3582873(2000)$.

12. Ice accretion modelling. URL http://www.tra3.com/icecremo/.

13. S.K. Thomas, R.P. Cassoni \& C.D. MacArthur, Aircraft anti-icing and de-icing techniques and modelling, Journal of Aircraft 33(5) pp841-854, (1996).

14. I.A. Frigaard, Solidification of spray formed billets, J. Engng Math. 31, 411 (1997).

15. E. Gutierrez-Miravete, E.J. Lavernia, G.M. Trapaga, J. Szekely \& N.J. Grant, Mathematical model of the spray deposition process, Metallurgica Transactions 20A, 71 (1989).

16. D.E. Kataoka \& S.M. Troian A theoretical study of instabilities at the advancing front of thermally driven coating flows, J. Coll. Interf. Sci. 192(2), 350 (1997).

17. D.E. Kataoka \& S.M. Troian "Stabilizing the advancing front of thermally driven climbing films," J. Coll. Interf. Sci. 203(2), 335 (1998).

18. S. Middleman "The effect of induced air-flow on the spin coating of viscous liquids," J. Appl. Phys. 62(6), 2530 (1987).

19. T.G. Myers \& D.W. Hammond "Ice and water film growth from incoming supercooled droplets," Int. J. Heat Mass Trans. 42, 2233 (1999).

20. K. Arnold, G. Tetzlaff \& A. Raabe "Modelling of ice accretion on a non-rotating cylinder" Meteorol. Zeitschrift 6(3), 120 (1997). 
21. T.G. Myers "An extension to the Messinger model for aircraft icing" AIAA J. 39(2), $211(2001)$.

22. P. Bartlett Development of a new model of ice accretion on aircraft, Proceedings of 9th International Workshop on Atmospheric Icing on Structures, Chester UK June (2000).

23. P.S. Hammond Nonlinear adjustment of a thin annular film of viscous fluid surrounding athread of another within a circular cylindrical pipe. J. Fluid Mech. 137, 363-384 (1983).

24. L.W. Schwartz, H.M. Princen \& A.D. Kiss On the motion of bubbles in capillary tubes. J. Fluid Mech. 172, 259-275 (1995).

25. S.G. Yiantsos \& B.G. Higgins Rayleigh-Taylor instability in thin viscous films. Phys. Fluids A(1) 1484-1501 (1989).

26. O.E. Jensen The thin liquid lining of a weakly curved cylindrical tube. J. Fluid Mech. 331, 373-403 (1997).

27. D.E. Weidner, L.W. Schwartz \& M.H. Eres Simulation of coating layer evolution and drop formation on horizontal cylinders. J. Colloid \& Interf. Sci. 187, 243-258 (1997).

28. D.E. Weidner, L.W. Schwartz \& R.R. Eley Role of surface tension gradients in correcting coating defects in corners. J. Colloid \& Interf. Sci. 179, 66-75 (1996).

29. Schwartz L.W. \& Weidner D.E. Modeling of coating flows on curved surfaces. J. Engng Math. 29 pp91-103 1995.

30. A.L. Frenkel Nonlinear theory of strongly undulating thin films flowing down vertical cylinders. Europhys. Lett. 18, 583-588 (1992).

31. S. Kalliadasis \& H-C Chang Drop formation during coating of vertical fibres. J. Fluid Mech. 261, 135-168 (1994).

32. E.B. Dussan V. \& S.H. Davis On the motion of fluid-fluid interface along a solid surface. J. Fluid Mech. 65 71-95 (1974). 
33. R.J. LeVeque. Numerical methods for conservation laws, Lectures in Mathematics, ETH Zürich, Birkhäuser (1990).

34. J.W. Thomas. Numerical partial differential equations: finite difference methods. Texts in Applied Mathematics (22), Springer (1995).

35. J.W. Thomas. Numerical partial differential equations: conservation laws and elliptic equations. Texts in Applied Mathematics (33), Springer (1995).

36. J.A. Moriarty \& L.W. Schwartz. Unsteady spreading of thin liquid films with small surface tension. Physics of Fluids A3(5), 733-742 (1991).

37. E.O. Tuck \& L.W. Schwartz. A numerical and asymptotic study of some third-order ordinary differential equations relevant to draining and coating flows. SIAM Rev. 32, 453-469 (1990).

38. M.H. Eres, D.E. Weidner \& L.W. Schwartz. Three-dimensional direct numerical simulation of surface-tension-gradient effects on the leveling of an evaporating multicomponent fluid. Langmuir 15(5), 1859-1871 (1999).

39. N. N. Yanenko. The method of fractional steps. Springer (1971).

40. J. Douglas Jr. Alternating directions methods for three space variables. Numerische Mathematik 4, 41-63 (1962).

41. A. Münch. Shock transitions in Marangoni gravity-driven thin-film flow. Nonlinearity $13,731-746(2000)$.

42. A.L. Bertozzi, A. Münch, X. Fanton \& A.M. Cazabat. Contact line stability and 'undercompressive shocks' in driven thin film flow. Physical Review Letters 81 (23), 5169-5172 (1998).

43. A.L. Bertozzi, A. Münch \& M. Shearer. Undercompressive shocks in thin film flows. Physica D 134, 431-464 (1999). 
44. J.P.F. Charpin. Water flow on accreting ice surfaces. Phd Thesis, Cranfield University, In preparation.

45. K.J. Finstad \& L. Makkonen Improved numerical model for wind turbine icing. Proc. 7th International Workshop on Atmospheric Icing of Structures, Quebec 1996.

46. W. Boehm. Bézier presentation of airfoils. Computer Aided Geometric Design 4(1), 17-22 (1987).

47. FLUENT 5 User's Guide, FLUENT Incorporated, Centerra Resource Park, 10 Cavendish Court, Lebanon NH03766,July 1998.

48. R.J. Kind, M.G. Potapczuk, A. Feo, C. Golia \& A.D. Shah. Experimental and computational simulation of in-flight icing phenomena. Progress in Aerospace Sciences 34, 257-345 (1998). 

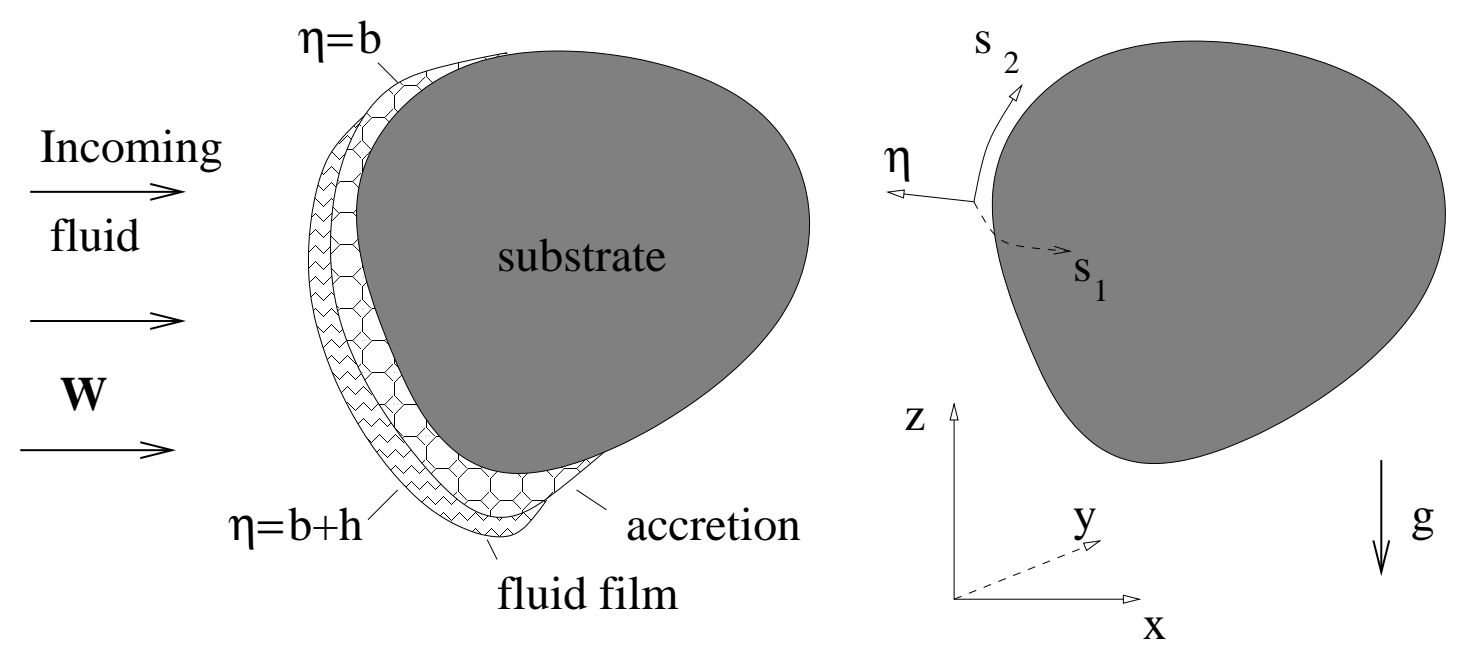

Figure 1: Configuration for solidification and fluid flow 


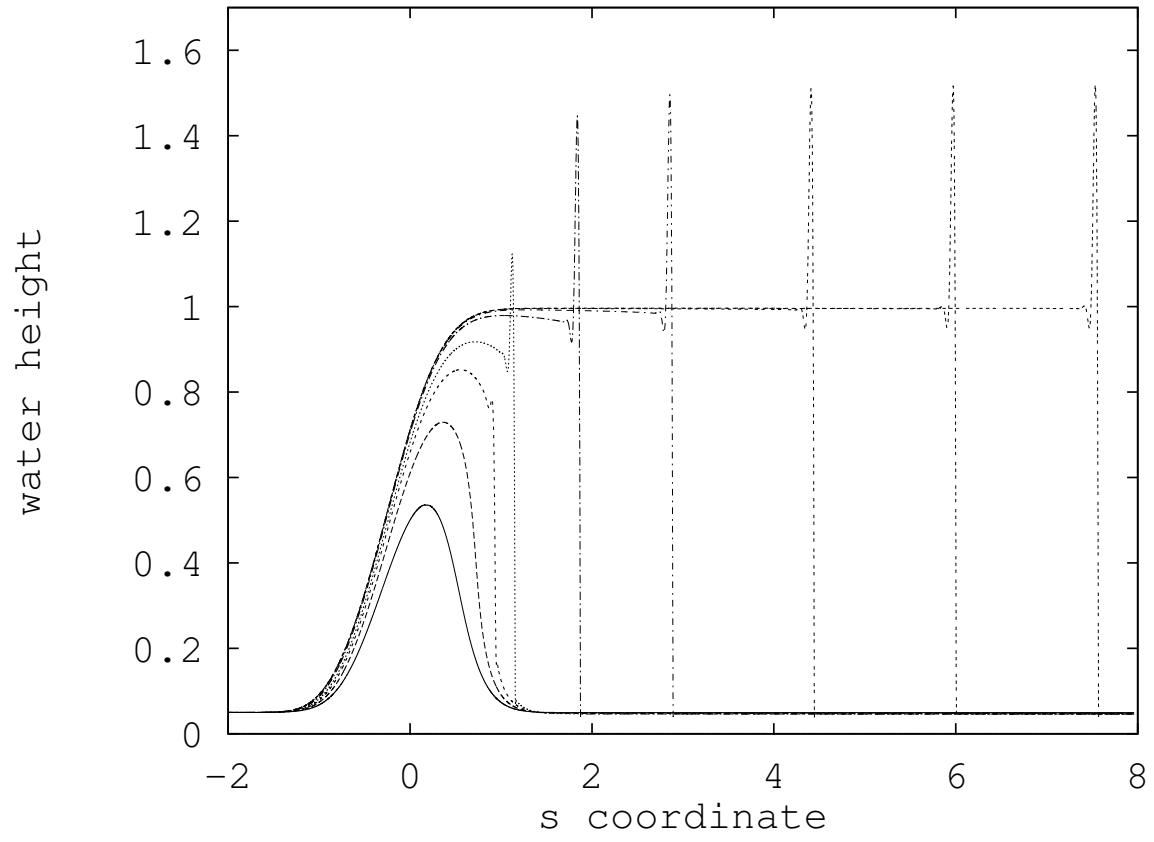

Figure 2: Two-dimensional fluid film driven by surface shear at times $t=1,3,4,4.5,5,7,9$, 11,15 


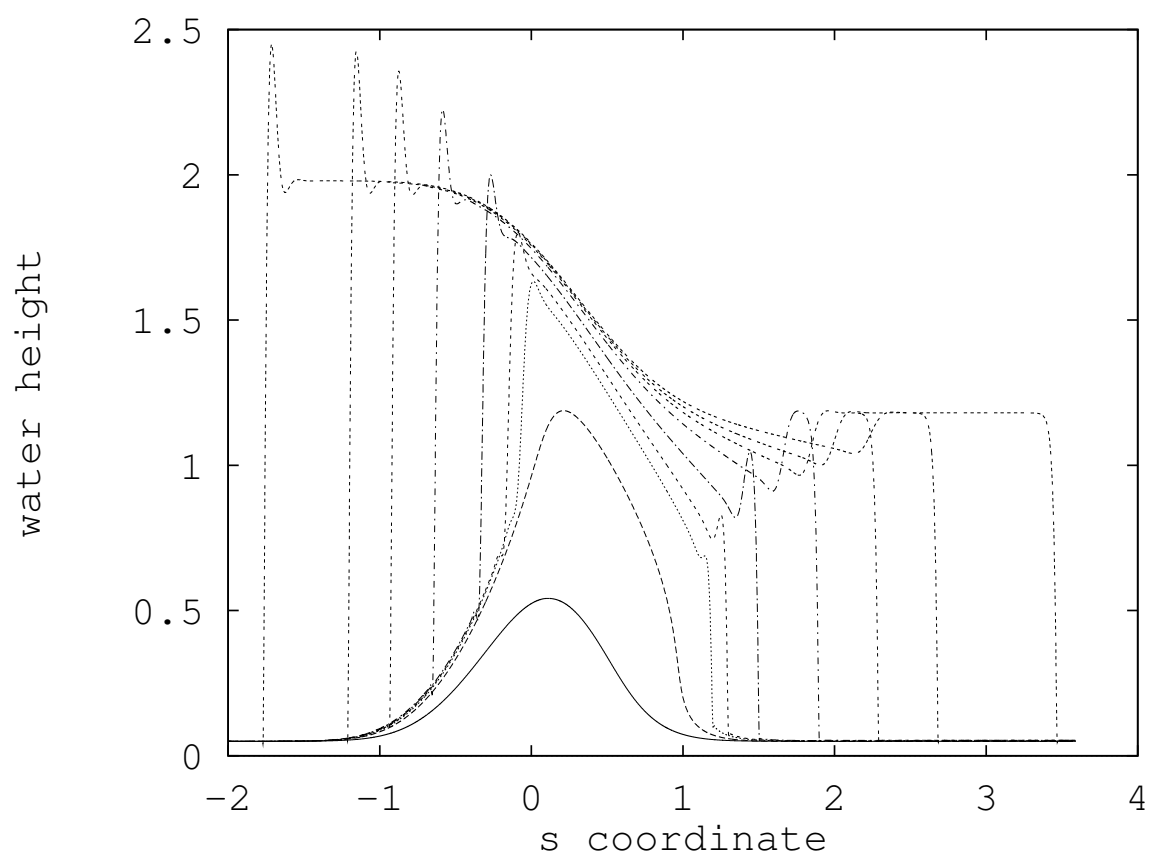

Figure 3: Two-dimensional fluid film driven by surface shear and gravity at times $t=1,3$, $4,4.5,5,7,9,11,15$ 


\section{film height}

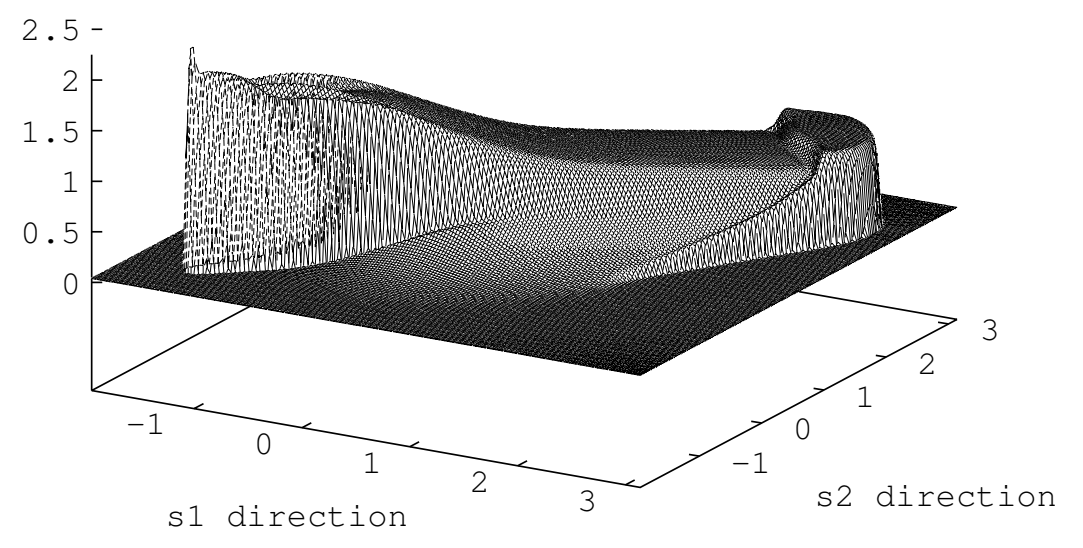

Figure 4: Three-dimensional fluid film driven by surface shear and gravity on a flat surface 

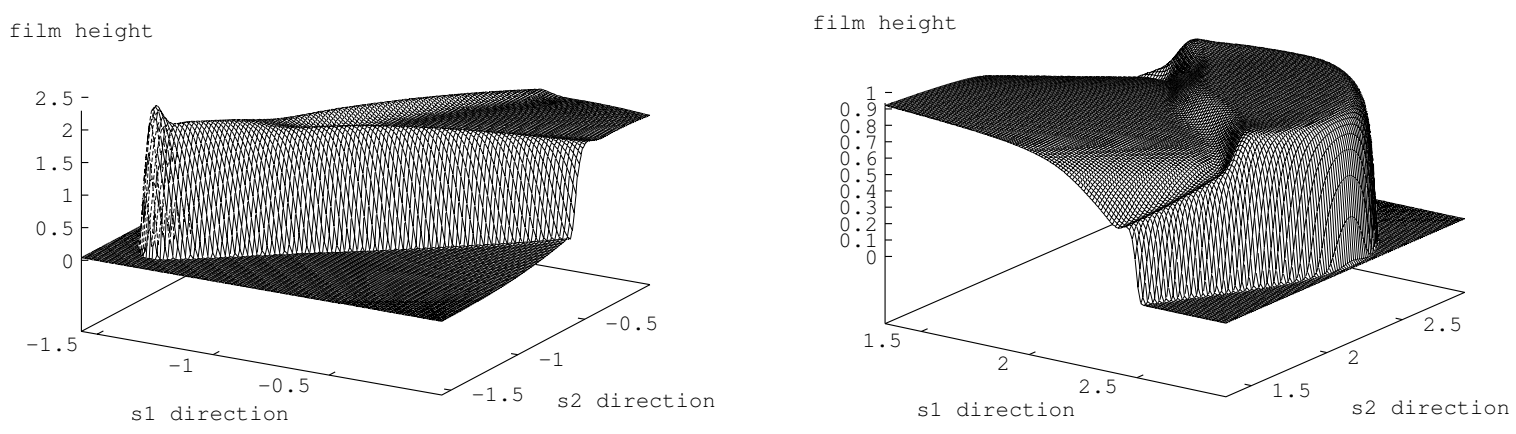

Figure 5: Close-up of moving fronts from Figure 4: (a) left hand front (b) right hand front 

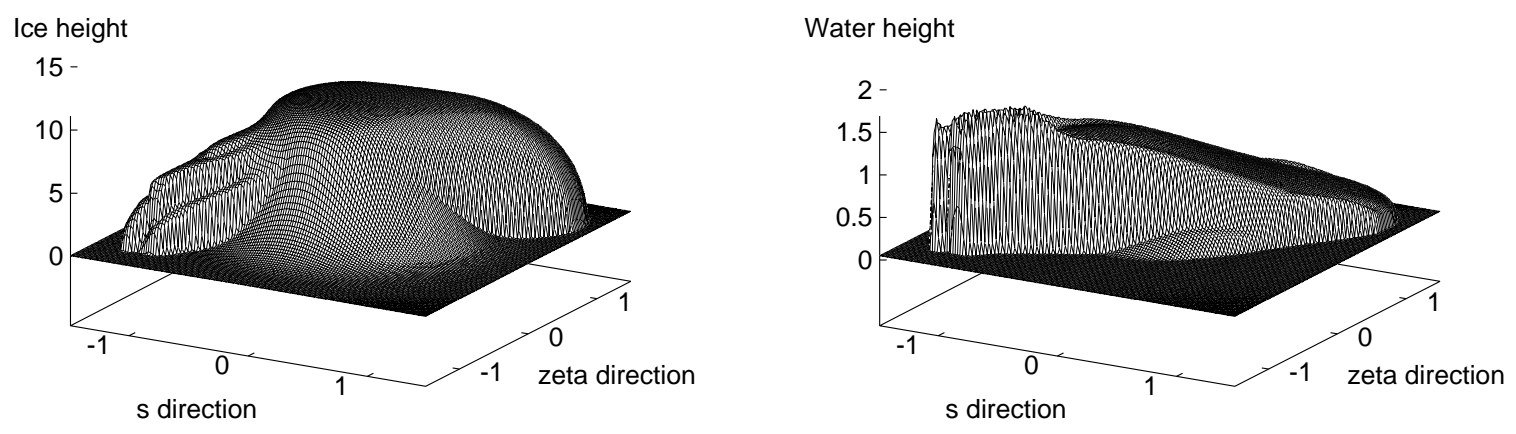

Figure 6: (a) Accretion thickness on a flat substrate at $t=60$

(b) corresponding film thickness 

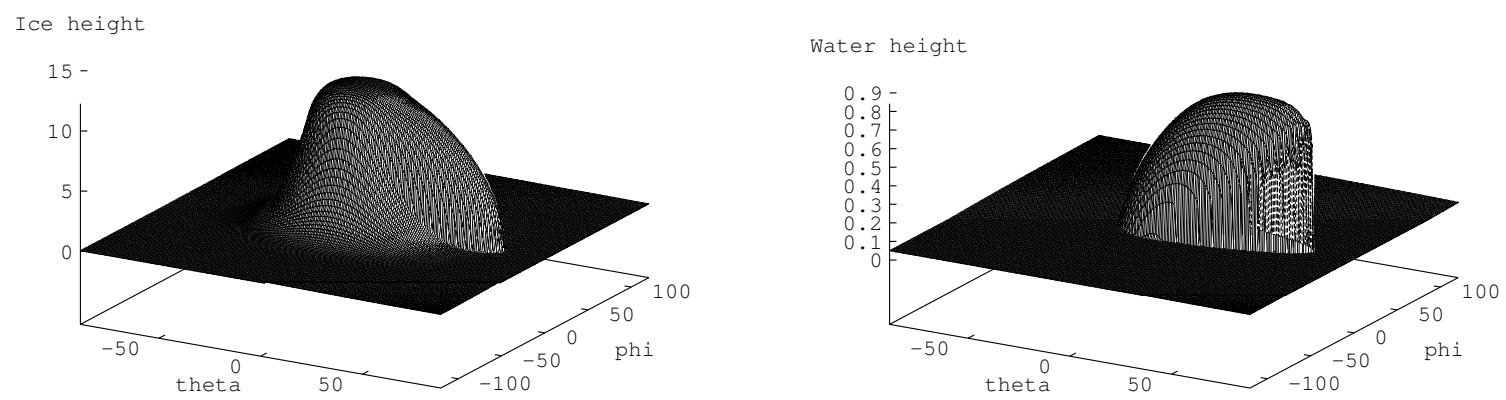

Figure 7: (a) Accretion thickness on a sphere at time $t=30$

(b) corresponding film thickness 


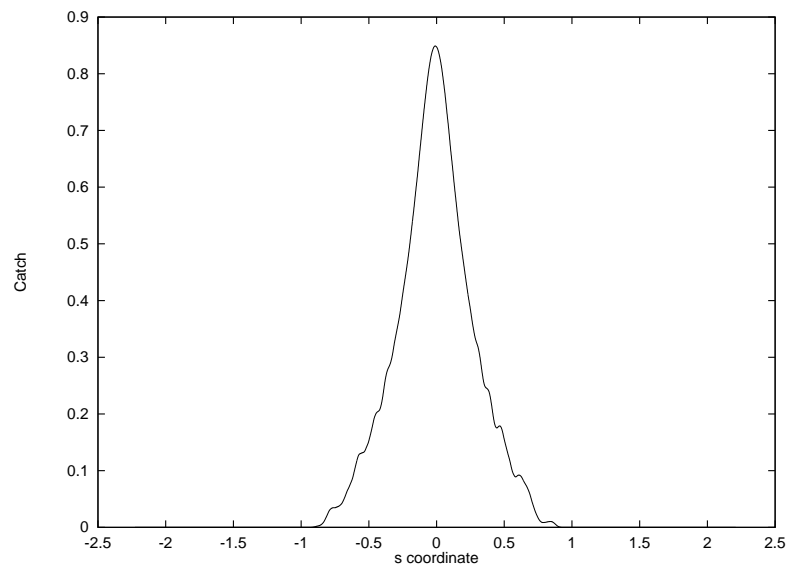

(a)

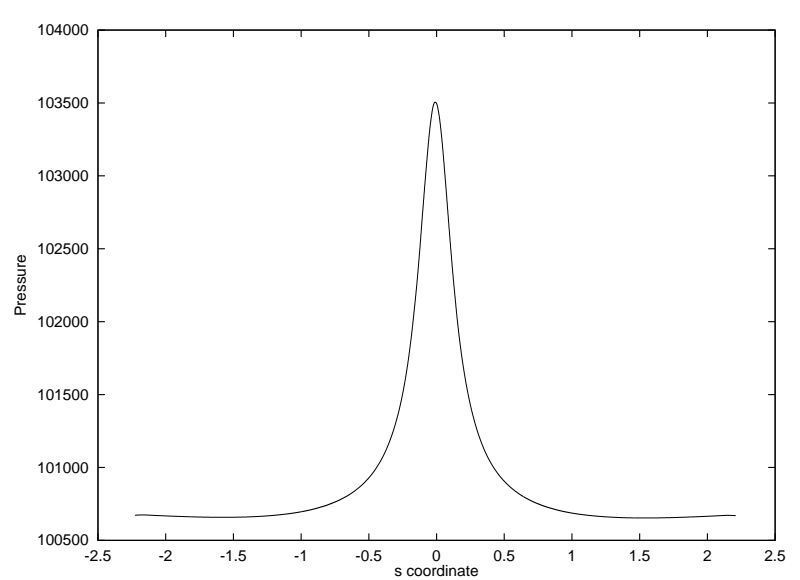

(c)

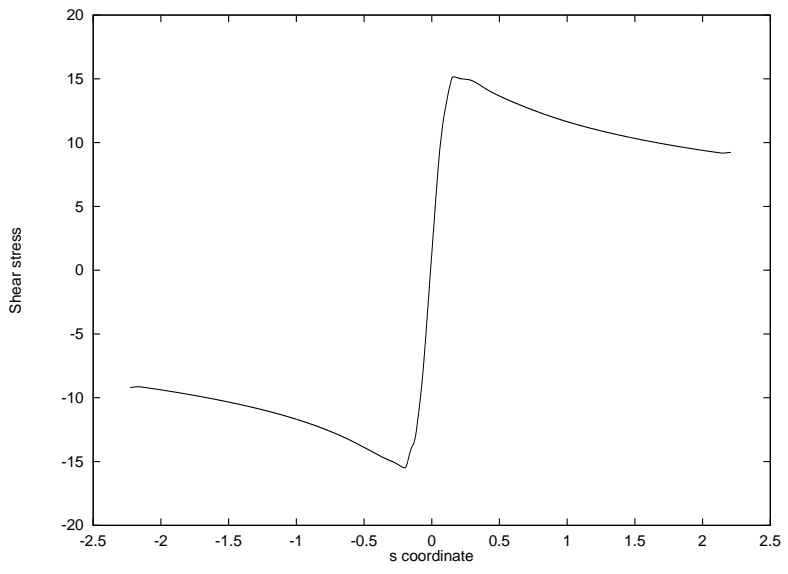

(b)

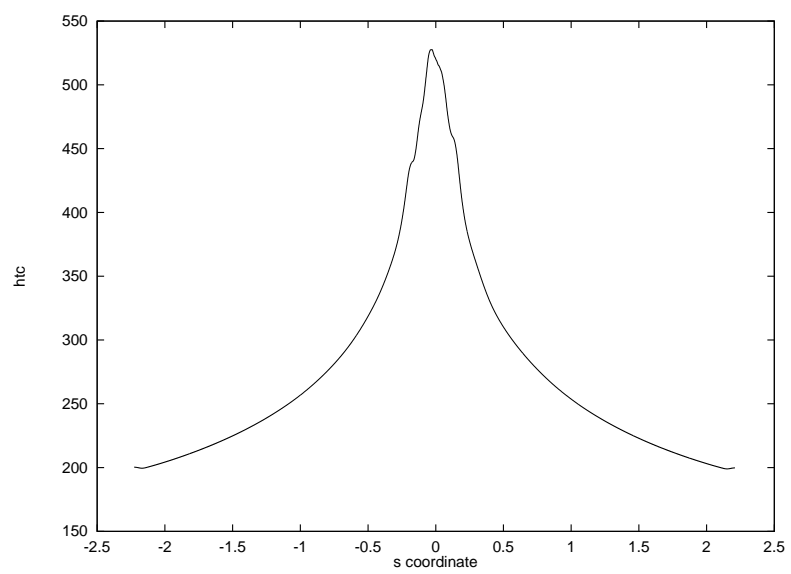

(d)

Figure 8: Flow around an aerofoil: (a) Collection efficiency (b) shear stress (c) pressure (d) heat transfer coefficient 


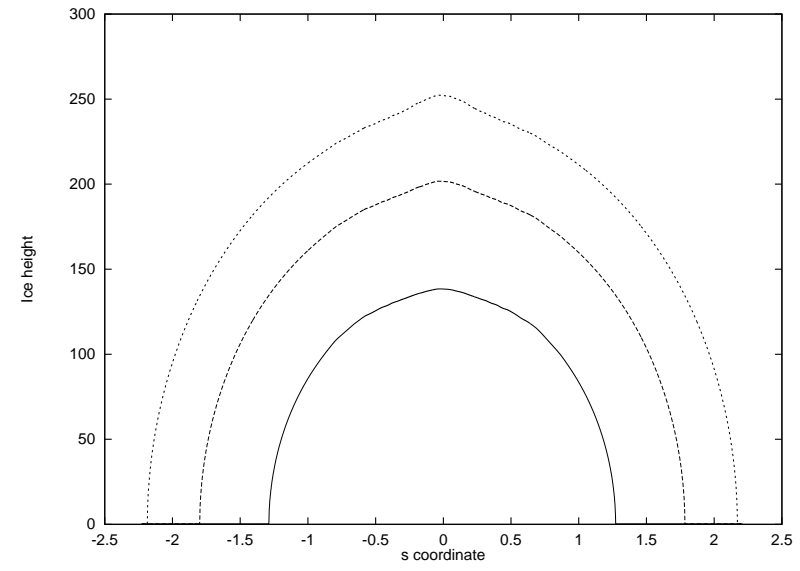

Figure 9: (a) Ice thickness on aerofoil at $t=500,1000,1500$ thickness

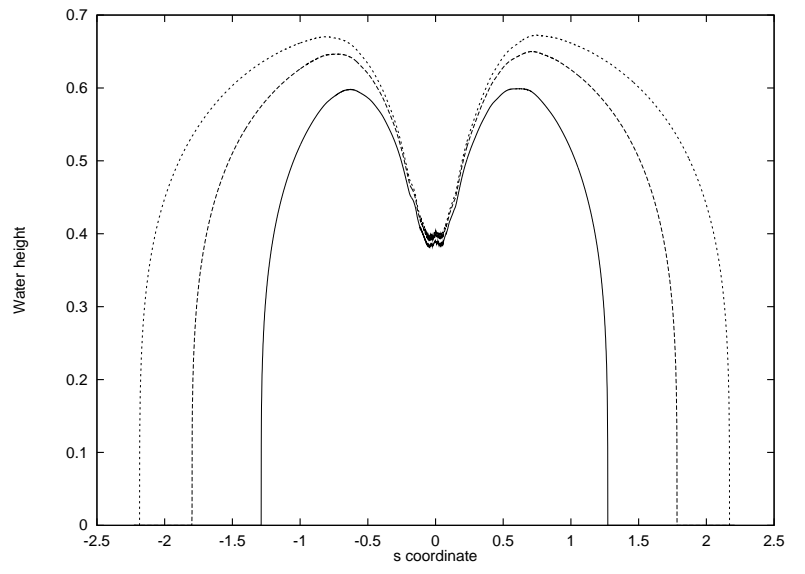

(b) corresponding water 